Mon. Not. R. Astron. Soc. 000,115(2007) Printed 30 October $2018 \quad$ (MN LATEX style file v2.2)

\title{
Supernova Kicks and Misaligned Be Star Binaries
}

\author{
Rebecca G. Martin, Christopher A. Tout and J. E. Pringle \\ University of Cambridge, Institute of Astronomy, The Observatories, Madingley Road, Cambridge CB3 OHA
}

\begin{abstract}
Be stars are rapidly spinning B stars surrounded by an outflowing disc of gas in Keplerian rotation. Be star/X-ray binary systems contain a Be star and a neutron star. They are found to have non-zero eccentricities and there is evidence that some systems have a misalignment between the spin axis of the star and the spin axis of the binary orbit. The eccentricities in these systems are be caused by a kick to the neutron star during the supernova that formed it. Such kicks would also give rise to misalignments. In this paper we investigate the extent to which the same kick distribution can give rise to both the observed eccentricity distribution and the observed misalignments. We find that a Maxwellian distribution of velocity kicks with a low velocity dispersion, $\sigma_{k} \approx 15 \mathrm{~km} \mathrm{~s}^{-1}$, is consistent with the observed eccentricity distribution but is hard to reconcile with the observed misalignments, typically $i \geqslant$ $25^{\circ}$. Alternatively a higher velocity kick distribution, $\sigma_{k}=265 \mathrm{~km} \mathrm{~s}^{-1}$, is consistent with the observed misalignments but not with the observed eccentricities, unless postsupernova circularisation of the binary orbits has taken place. We discuss briefly how this might be achieved.
\end{abstract}

Key words: stars: emission lines, Be; stars: neutron; X-rays: binaries; accretion, accretion discs; supernovae

\section{INTRODUCTION}

Be stars were discovered by Secchi (1867) who observed emission lines in $\gamma$ Cas. These stars are rapidly rotating at about 70 per cent of their break up velocity (Porter 1996). In fact, relative to their break up velocities, they are the fastest rotating bodies observed. They are early type main-sequence stars which have shown $\mathrm{H} \alpha$ in emission at least once. They are variable in brightness and spectra which show broad HeI absorption and emission at either visual or UV wavelengths. It is found that the emission, and so presumably the discs, are only temporary and so Be stars become B stars and vice versa.

If the disc is viewed edge on the Be star is seen as a shell star. The spectra then show Balmer emission with sharp absorption cores, narrow absorption lines of ionized metals and broad HeI absorption. Be-star discs vanish and re-appear on timescales of a few hundred days. Dachs, Kiehling \& Engels (1988) studied Balmer emission line profiles and concluded that the envelopes surrounding Be stars are in Keplerian motion within the disc. The disc shows optical and IR emission lines and an IR continuum excess.

The stars $\gamma$ Cas and 59 Cyg have shown two successive shell events. These were associated with a remarkably synchronous quasi-cyclic variation of the emission line width in all observed emission lines that has been called spectacular variation (Hummel 1998). The change in emission line width removes the correlation between the projected surface velocity, $v \sin i$, and the FWHM (full width half maximum) and so a circumstellar equatorial disc fails to explain the spectacular variations. The emission lines and shell Be stars are explained by differences in disc inclination to the line of sight, so transitions between the two were not expected. Hummel (1998) explains the spectacular variations by a Keplerian disc which is somehow tilted with respect to the equatorial plane of the star. The variation in emission line widths and profile shapes are then due to the precession of the disc. He suggests that the sequence of alternating shell-phases and narrow single peak phases is due to the variation in disc inclination caused by precession. The idea that a disc might change its inclination to the line of sight is borne out by observations of 28 Tau (Pleione) by Hirata (2007). This star also changes between B star, Be star and shell star and in this star the intrinsic polarization angle changes in phase with these variations. The cause of the precession is also not clear but Hummel (1998) suggested that it might be induced by tides from a binary companion. The two systems $\gamma$ Cas and 59 Cyg are binary. The system 28 Tau appears to show radial velocity variations, although there is no confirmed orbital period (Rivinius, Sttefl \& Baade 2006). In both $\gamma$ Cas and 28 Tau the misalignment angle between the stellar equa- 
tor and the disc/orbital plane is thought to be around $25^{\circ}$ (Hummel 1998; Hirata 2007).

In further support of this possibility, we note that the Bstar binary PSR J0045-7319 has a spin-orbit misalignment suggested by its orbital plane precession Kaspi et al. 1996; Lai, Bildsten \& Kapsi 1995). This misalignment in a B-star orbit means that misalignment in Be stars in not uncommon. In this case the B star rotates retrogradely with respect to the orbit (Lai 1996a).

The standard model for Be-star discs is that they are decretion discs with the mass expelled from the neighbourhood of the Be star itself (Cassinelli et al. 2002). In this case we expect the plane of the inner disc to be aligned with the spin axis of the Be star. There are two reasons for the disc to be found at an angle discussed in the literature. First Porter (1998) suggested that the disc warping and precession might be caused by a radiation-induced instability (Pringle 1996). Secondly, as we reported above, it is widely suggested that the warp and precession are caused by a misalignment between the spin axis of the Be star and the orbit of the binary companion. If the disc is a decretion disc we expect the inner edge to be aligned with the equatorial plane of the $\mathrm{B}$ star and the outer edge to tend to be tidally aligned with the orbital plane. Thus there must be a warp at some radius in the disc. Here we focus on this second possibility and concentrate on the $\mathrm{Be} / \mathrm{X}$-ray binaries in which the companion stars are neutron stars.

Neutron stars, observed as radio pulsars, have space velocities much greater than their progenitors (Gunn \& Ostriker 1970). The accepted explanation for this is that supernova explosions are asymmetric and give very large kicks to the newly formed neutron stars (Shklovskii 1970; Sutantyo 1978). Indeed some supernova remnants show evidence for asymmetric explosions (Morse, Winkler \& Kirshner 1995; Aschenbach. Egger \& Trumper 1995). Thus any system that contains a neutron star could have had a supernova kick. It is also found that Be/X-ray binaries have high eccentricities that cannot be explained without supernova kicks (Verbunt \& van der Heuvel 1995).

In a Be-star binary system, prior to the supernova in which the core of its companion collapses to a neutron star, we expect the Be-star spin to aligned with the orbit and for the orbit to be circular. The kick from the supernova has two effects, it makes the orbit eccentric, and perhaps even unbinds it, and it misaligns the orbit with the spin axis of the Be star. Thus information about the distribution of eccentricities in Be-star systems can in principle give us information about the distribution of spin-orbit misalignments.

Lyne \& Lorimer (1994) analysed the known pulsar velocities and concluded that they were born with a mean speed of about $450 \mathrm{~km} \mathrm{~s}^{-1}$. Hansen \& Phinney (1997) considered the selection effects, as a result of the flux limits, of the pulsar surveys and the accuracy of the proper motion determinations and found a mean birth speed of around $250-300 \mathrm{~km} \mathrm{~s}^{-1}$. This is consistent with a Maxwellian distribution with $\sigma_{\mathrm{k}}=190 \mathrm{~km} \mathrm{~s}^{-1}$ which has a mean velocity of $\left\langle v_{k}\right\rangle=303 \mathrm{~km} \mathrm{~s}^{-1}$. More recently Arzoumanian, Chernoff \& Cordes (2002) found a best fitting distribution with two Maxwellian components, one for
40 per cent of the pulsars with $\sigma_{\mathrm{k} 1}=90 \mathrm{~km} \mathrm{~s}^{-1}$ and the other with $\sigma_{\mathrm{k} 2}=500 \mathrm{~km} \mathrm{~s}^{-1}$.

Subsequently Hobbs et al. (2005) extended the work of Lvne \& Lorimer (1994) with a much larger sample of single radio pulsars and claimed that the kick distribution is consistent with a single Maxwellian with $\sigma_{\mathrm{k}}=265 \mathrm{~km} \mathrm{~s}^{-1}$ without a significant low-velocity component. That such a kick distribution cannot reproduce the period-eccentricity distribution of Be/X-ray binaries has already been noticed and discussed by Pfahl et al. (2002) and van den Heuvel (2007). They concluded that a bimodal kick distribution is needed with a low-velocity component $\left(\sigma_{\mathrm{k}}<50 \mathrm{~km} \mathrm{~s}^{-1}\right)$ when the supernova occurs in a binary system. The idea that there might be two types of collapse and therefore two types of supernova kick was originally proposed by Katz (1975). Podsiadlowski et al. (2004) also find evidence for a two-component kick distribution with low-velocity kicks mainly in close binaries. Kramer \& Stairs (2008) give an extensive discussion of the second kick in the double pulsar J0737-3039 and conclude that it probably had to be small. Theoretical studies such as those by Scheck et al. (2006) and Kitaura, Janke \& Hillebrandt (2006) do not yet throw much light on this but it may be that there are more lower mass supernovae, with smaller kicks, in binary systems or that the binary orbit quenches the hydrodynamic instabilities which lead to a very asymmetric explosion.

In a binary system, if the kick is too strong, the system does not remain bound. Even a small velocity kick can lead to a large eccentricity and inclination between the old and new orbits (Brandt \& Podsiadlowski 1995). We do not know how the angular momentum of the remnant is also affected by the supernova kick so we do not know how much of the misalignment of the neutron star now is caused by the orbital inclination. We can however expect the companion to continue spinning aligned with the pre-supernova orbit immediately after the explosion. There are several binary systems with neutron star companions that are observed to be misaligned.

Brandt \& Podsiadlowski 1995) investigated some of the effects of high supernova kick velocities on the orbital parameters of post-supernova neutron-star binaries. Here we look at a variety of velocity kick distributions and consider the implications for the distribution of the inclinations between the orbit before and after the kick. After ensuring that we can reproduce the work of Brandt \& Podsiadlowski (1995) we model Be-star systems with our preferred distributions for their progenitors including a range of masses. There is somewhat more data now available for comparison in the period-eccentricity plane and we find that, though all systems can be formed with our models, they tend to be more circular than expected with kicks distributed according to Hobbs et al. (2005). We investigate what kick distribution could lead to the observed eccentricity distribution and also how the eccentricity distribution might have changed since the supernova. We consider three types of kick distribution, a single peaked Maxwellian velocity kick distribution, kicks which are direction limited and a double Maxwellian distribution. 


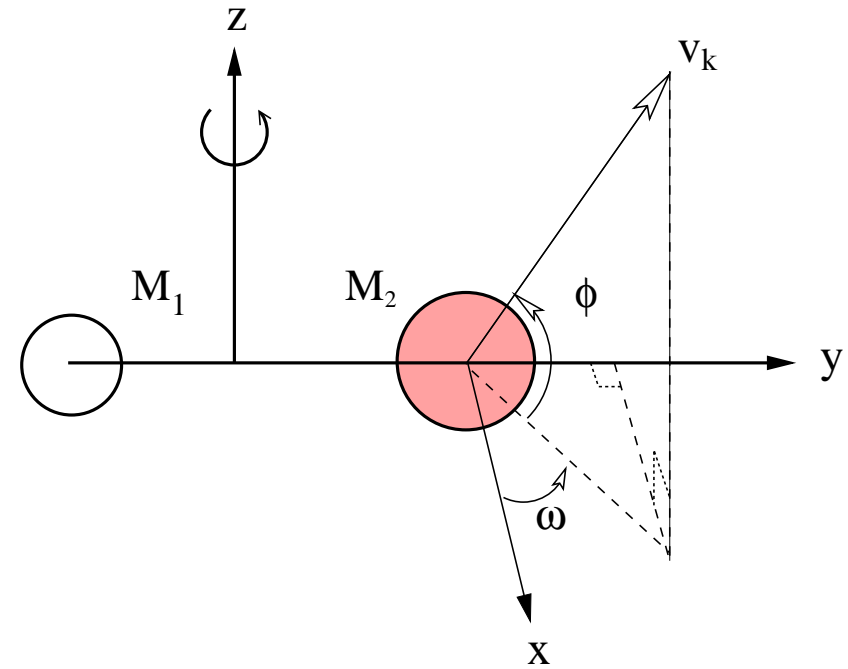

Figure 1. The system before the supernova. The two stars of mass $M_{1}$ and $M_{2}$ are in a circular orbit about their centre of mass at the origin. The orbital angular momentum is in the $z$ direction and the orbit is in the $x y$-plane. When star 2 explodes as a supernova it is travelling in the direction of the negative $x$-axis with speed $v_{\text {orb }}$ relative to star 1 . It receives a kick of velocity $v_{\mathrm{k}}$ at an angle $\phi$ to the plane of the binary orbit. The angle between the projection of the velocity kick on to the binary plane and the $x$-direction is $\omega$.

\section{MISALIGNMENT PROBABILITY DISTRIBUTION}

In this section we consider the effect of a velocity kick on the orbital inclination. We start with a binary in a circular orbit. One star then has an asymmetric supernova explosion which gives it a kick with velocity $0 \leqslant v_{\mathrm{k}}<\infty$ in a direction given by the angle $\phi$ out of the binary plane $(-\pi / 2 \leqslant \phi \leqslant$ $\pi / 2$ ) and an angle between the direction opposite to the instantaneous velocity of the star and the projection of the velocity kick into the binary orbital plane of $0 \leqslant \omega<2 \pi$ (see Fig. 1). For now we assume that no mass is lost.

We are interested in the misalignment angle of the system, $i$, after the supernova kick. This is the angle between the old and new angular momenta of the orbits (Fig. 2). If $0 \leqslant i<\pi / 2$ then the system is closer to alignment than counter-alignment and if $\pi / 2<i \leqslant \pi$ it is closer to counteralignment. Brandt \& Podsiadlowski (1995) find this angle to be given by

$$
\cos i=\frac{v_{\mathrm{orb}}-v_{\mathrm{k}} \cos \omega \cos \phi}{\left[v_{\mathrm{k}}^{2} \sin ^{2} \phi+\left(v_{\mathrm{orb}}-v_{\mathrm{k}} \cos \omega \cos \phi\right)^{2}\right]^{\frac{1}{2}}},
$$

where $v_{\text {orb }}$ is the initial orbital velocity of the system. The relative velocity of the stars after the supernova is

$$
v_{\mathrm{n}}^{2}=v_{\mathrm{k}}^{2}+v_{\mathrm{orb}}^{2}-2 v_{\text {orb }} v_{\mathrm{k}} \cos \omega \cos \phi .
$$

We can rearrange equation (1) to find

$$
\cos \omega=\frac{v_{\text {orb }}}{v_{\mathrm{k}}} \frac{1}{\cos \phi} \pm \frac{\tan \phi}{\tan i}
$$

when $v_{\mathrm{k}} \neq 0, \tan i \neq 0$ (so that $i \neq 0, \pi$ ) and $\cos \phi \neq 0$ (so that $\phi \neq-\pi / 2, \pi / 2$ ). Then if $0 \leqslant i<\pi / 2$ from equation (1) we have $v_{\text {orb }}>v_{\mathrm{k}} \cos \omega \cos \phi$. With equation (3) this

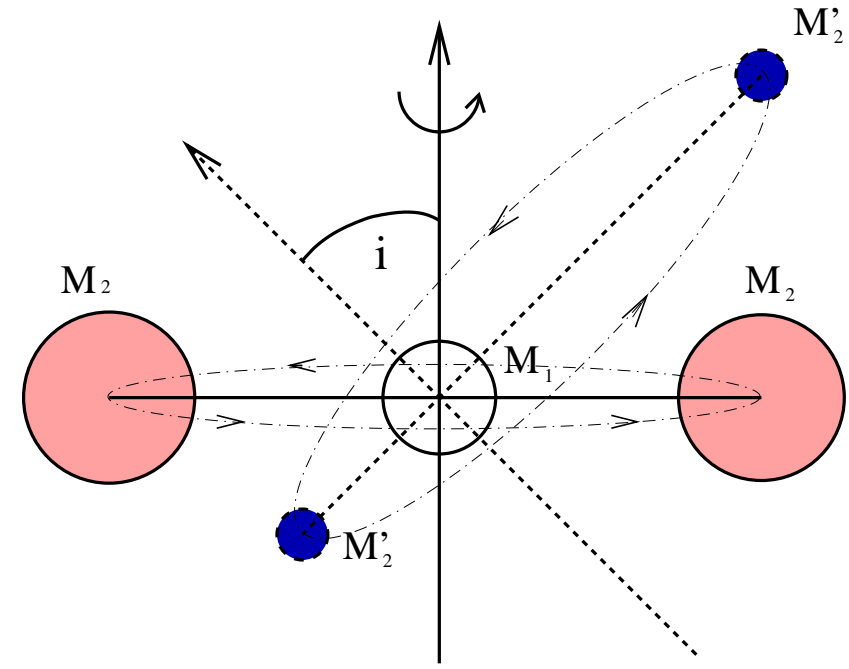

Figure 2. The binary system before and after the supernova in the frame of the star 1 of mass $M_{1}$. The mass of star 2 is reduced from $M_{2}$ to $M_{2}^{\prime}$ in the supernova and it experiences the kick. The straight solid arrow is the orbital angular momentum of the system before the supernova. The other solid straight line is the pre-supernova diameter of the circular orbit indicated by the dot-dashed ellipse. The dashed arrow is the orbital angular momentum after the supernova. The angle $i$ is the angle of misalignment between the pre- and post-supernova orbits. If star 1 has its spin aligned with the pre-supernova orbit, then the angle $i$ also measures the misalignment between the post-supernova eccentric orbit and the spin of star 1 . The other dashed line is the major axis of the post-supernova orbit indicated by a dot-dash line.

corresponds to

$$
\mp \frac{\tan \phi}{\tan i} v_{\mathrm{k}} \cos \phi>0
$$

and because $\tan i>0$ we see

$$
\mp \sin \phi>0 \text {. }
$$

Similarly when $\pi / 2<i \leqslant \pi$, so that $\tan i<0$, we find the same condition as above on $\sin \phi$. Now we can rewrite equation (3) as

$$
\cos \omega=\frac{v_{\mathrm{orb}}}{v_{\mathrm{k}}} \frac{1}{\cos \phi}-\frac{|\tan \phi|}{\tan i}
$$

We consider where this equation has real valued solutions in the $\phi-v_{\mathrm{k}}$ plane. In Fig. 3 we plot the locus of $\cos \omega=1$ $(\omega=0)$,

$$
v_{+}=\frac{v_{\text {orb }}}{\cos \phi}\left(1+\frac{|\tan \phi|}{\tan i}\right)^{-1}
$$

and the locus of $\cos \omega=-1(\omega=\pi)$,

$$
v_{-}=\frac{v_{\text {orb }}}{\cos \phi}\left(-1+\frac{|\tan \phi|}{\tan i}\right)^{-1},
$$

for four values of $i$. The region between the $v_{+}$and $v_{-}$contours is the region where we have real values of $\cos \omega$ and it represents the combinations of kick parameters which can lead to a misalignment of the chosen $i$. 

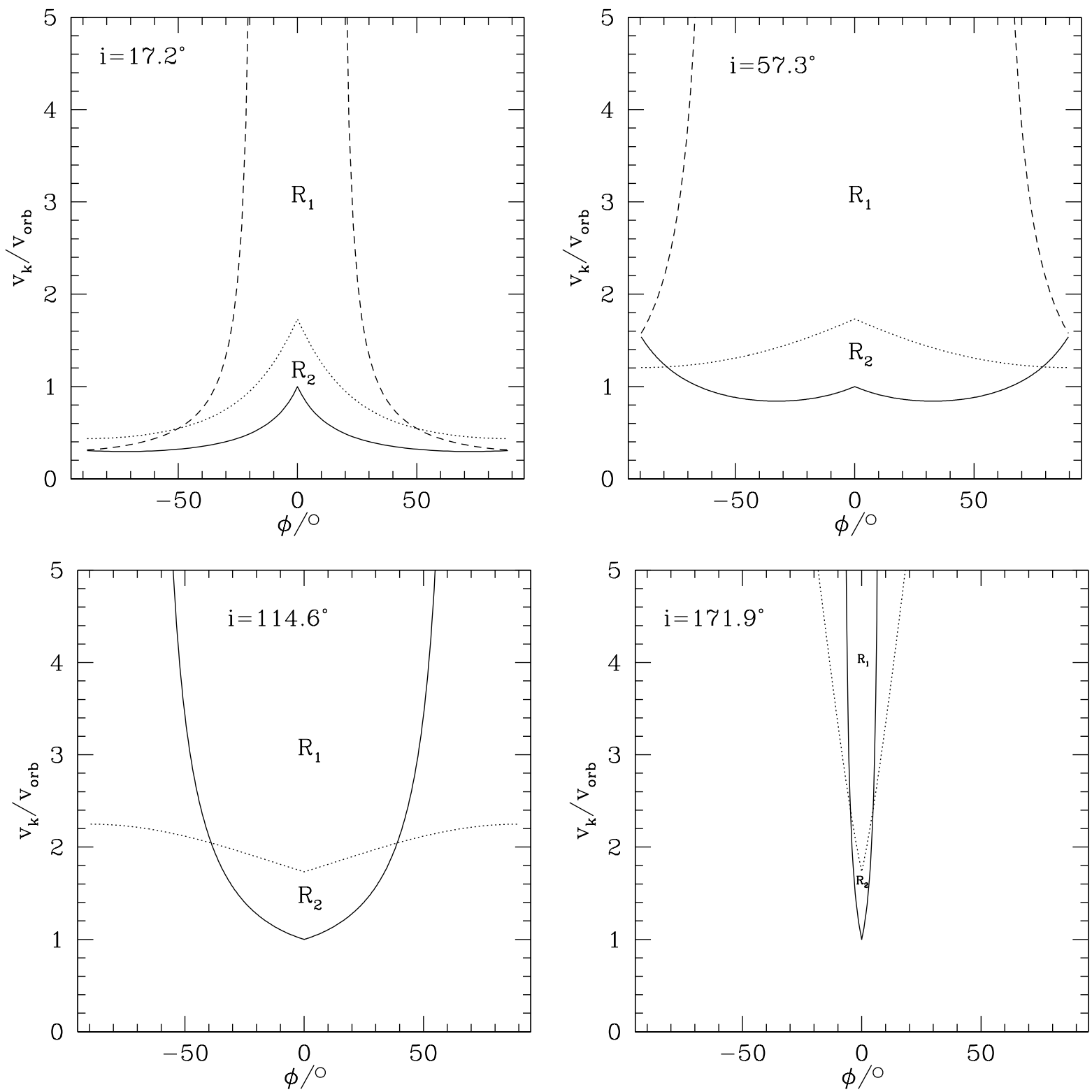

Figure 3. Possible combinations of the ratio of star 2's supernova kick velocity, $v_{\mathrm{k}}$, to the relative orbital velocity, $v_{\mathrm{orb}}$ and angle $\phi$ between the kick direction and the orbital plane for four different inclinations $i$ between the pre- and post-supernova orbital planes, top left $i=17.2^{\circ}$, top right $i=57.3^{\circ}$, bottom left $i=114.6^{\circ}$ and bottom right $i=171.9^{\circ}$. For angles $i>90^{\circ}$ the post-supernova orbit counter rotates with respect to the spin of star 1 . For each value of $\phi$ there is a range of values of $v_{\mathrm{k}}$ which can give rise to the required misalignment $i$. This depends on the angle $\omega(0 \leqslant \omega<2 \pi)$ shown in Fig. 1 In each panel, the solid line corresponds to $\omega=0$ (and thus to the kick velocity $v_{+}$, equation 7 ) and the dashed line to $\omega=\pi$ (kick velocity $v_{-}$, equation 8 ). Note that $\omega=0, \phi=0$ corresponds to a kick directly opposed to the motion of star 2 and $\omega=\pi, \phi=0$ corresponds to a kick in the direction of motion of star 2 . In the bottom two plots, which correspond to post-supernova retrograde motion, it is not possible to achieve this with a prograde kick $(\omega=\pi)$ for any value of $\phi$ so that the dashed lines corresponding to $\omega=\pi$ are absent. The dotted lines show the maximum velocity kick, $v_{\text {bound }}$, (equation 26) as a function of $\phi$, for which the system remains bound. Below this line the system remains bound after the supernova and above it the binary is disrupted. In order to find the probability distribution $P(i)$ of the misalignment angle $i$ in equation (18) we integrate in $\left(v_{\mathrm{k}} / v_{\mathrm{orb}}, \phi\right)$-space over the regions $R=R_{1}+R_{2}$ between the contours of $v_{+}$(solid line), $v_{-}$(dashed line). To find only the probability distribution $P(i)$ for the bound systems alone we integrate over the regions $R_{2}$ only.

\section{ISOTROPIC MAXWELLIAN KICK DISTRIBUTION}

As an illustration we apply these results to a simple isotropic Maxwellian kick distribution. For an isotropic kick distribution the direction of the kick velocity is uniformly dis- tributed over a sphere so that the angles defined in Fig. 1 are distributed as

$$
P(\omega) d \omega=\frac{1}{2 \pi} d \omega
$$


and

$$
P(\phi) d \phi=\cos \phi d \phi .
$$

We here choose the kick speed to have a Maxwellian distribution so that

$$
P\left(v_{\mathrm{k}}\right) d v_{\mathrm{k}}=\sqrt{\frac{2}{\pi}} \frac{v_{\mathrm{k}}^{2}}{\sigma_{\mathrm{k}}^{3}} e^{-\frac{v_{\mathrm{k}}^{2}}{2 \sigma_{\mathrm{k}}^{2}}} d v_{\mathrm{k}}
$$

where $\sigma_{\mathrm{k}}$ is the dispersion of the velocity, and recall that Hobbs et al. (2005) find $\sigma_{\mathrm{k}}=265 \mathrm{~km} \mathrm{~s}^{-1}$. In sections 5.2 and 5.3 we shall consider alternative velocity kick distributions. Because $i=i\left(v_{\mathrm{k}}, \phi, \omega\right)$ its probability distribution is

$$
P(i) d i=\int_{v_{\mathrm{k}}=0}^{\infty} \int_{\phi=-\frac{\pi}{2}}^{\frac{\pi}{2}} \int_{\omega=0}^{2 \pi} P(\phi) P(\omega) P\left(v_{\mathrm{k}}\right) d \omega d \phi d v_{\mathrm{k}} .
$$

We change variables from $\left(v_{\mathrm{k}}, \phi, \omega\right)$ to $\left(v_{\mathrm{k}}, \phi, i\right)$ and find

$$
P(i) d i=\int_{i}^{i+d i} \iint_{R} P(\phi) P(\omega) P\left(v_{\mathrm{k}}\right)|J| d \phi d v_{\mathrm{k}} d i,
$$

where $R=R_{1}+R_{2}$ is the region in the $\left(\phi, v_{\mathrm{k}}\right)$ plane where $\cos \omega$ is real valued. This is illustrated in Fig. 3 for different values of $i$. Outside of the region bounded by these curves, the given velocity kick and angle $\phi$ cannot produce a system misaligned by $i$ because then $|\cos \omega|>1$. We consider this region for bound systems in Section 3.1. The Jacobian, $J$, for the change of variables is given by

$$
d v_{\mathrm{k}} d \phi d \omega=|J| d v_{\mathrm{k}} d \phi d i
$$

where

$$
J=\left|\begin{array}{lll}
\left(\frac{\partial v_{\mathrm{k}}}{\partial v_{\mathrm{k}}}\right)_{\phi, i} & \left(\frac{\partial v_{\mathrm{k}}}{\partial \phi}\right)_{v_{\mathrm{k}}, i} & \left(\frac{\partial v_{\mathrm{k}}}{\partial i}\right)_{v_{\mathrm{k}}, \phi} \\
\left(\frac{\partial \phi}{\partial v_{\mathrm{k}}}\right)_{\phi, i} & \left(\frac{\partial \phi}{\partial \phi}\right)_{v_{\mathrm{k}}, i} & \left(\frac{\partial \phi}{\partial i}\right)_{v_{\mathrm{k}}, \phi} \\
\left(\frac{\partial \omega}{\partial v_{\mathrm{k}}}\right)_{\phi, i} & \left(\frac{\partial \omega}{\partial \phi}\right)_{v_{\mathrm{k}}, i} & \left(\frac{\partial \omega}{\partial i}\right)_{v_{\mathrm{k}}, \phi}
\end{array}\right| .
$$

Because $\omega, \phi$ and $v_{\mathrm{k}}$ are independently distributed we find

$$
J=\left|\begin{array}{ccc}
1 & 0 & 0 \\
0 & 1 & 0 \\
0 & 0 & \left(\frac{\partial \omega}{\partial i}\right)_{v_{\mathrm{k}}, \phi}
\end{array}\right|=\left(\frac{\partial \omega}{\partial i}\right)_{v_{\mathrm{k}}, \phi} .
$$

We differentiate equation (6) to find

$$
J=\left(\frac{\partial \omega}{\partial i}\right)_{v_{\mathrm{k}}, \phi}=-\frac{|\tan \phi|}{\sin \omega \sin ^{2} i} .
$$

Now we can write down the full probability density distribution for the misalignment as an integral

$$
P(i) d i=\sqrt{\frac{2}{\pi^{3}}} \frac{1}{2 \sigma_{k}^{3}} \int_{i}^{i+d i} \iint_{R} I d \phi d v_{\mathrm{k}} d i,
$$

where

$$
I=v_{\mathrm{k}}^{2} e^{-\frac{v_{\mathrm{k}}^{2}}{2 \sigma_{k}^{2}}} \frac{|\sin \phi|}{|\sin \omega| \sin ^{2} i}
$$

and $\omega=\omega\left(i, v_{\mathrm{k}}\right)$ is defined by equation (6).

This can now be integrated numerically for specific values of $v_{\mathrm{orb}} / \sigma_{\mathrm{k}}$ to find the probability distribution of the misalignment angle, $i$, of the system. We plot this as the solid lines in Fig. 团 for $v_{\text {orb }}=\sigma_{\mathrm{k}}$ (upper line on $y$-axis) and $v_{\text {orb }}=0.5 \sigma_{\mathrm{k}}$. The higher the misalignment angle, $i$, the lower its probability of forming. The higher $v_{\text {orb }} / \sigma_{\mathrm{k}}$, the

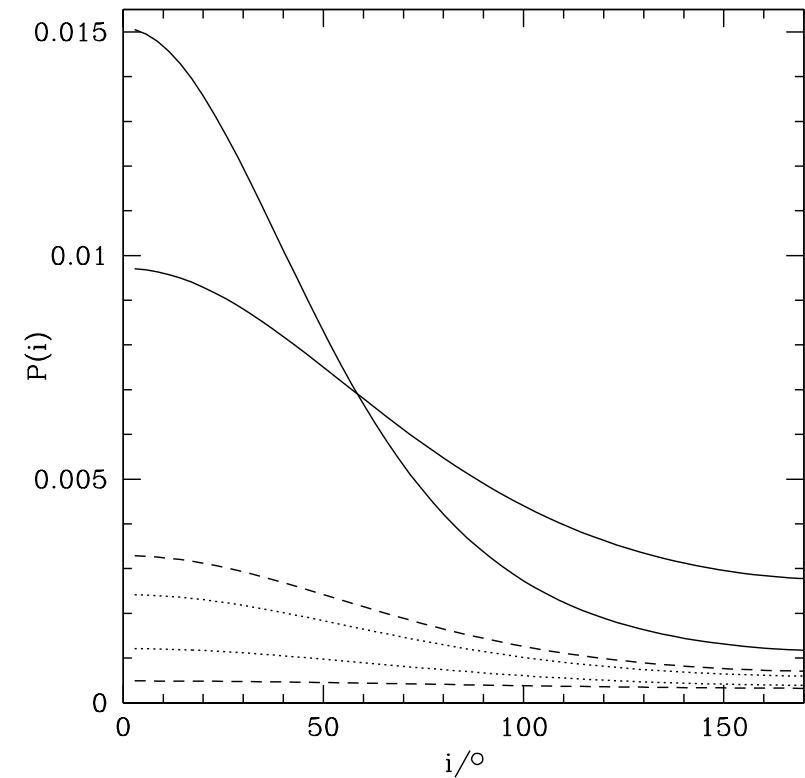

Figure 4. The probability distribution $P(i)$ of the postsupernova misalignment angle $i$ is plotted as a function of $i$ for the case when the probability distribution of the kick velocity $v_{\mathrm{k}}$ is a Maxwellian with dispersion $\sigma_{\mathrm{k}}$ and no mass is lost from the system $(f=0)$. The solid lines correspond to integrating over the full area $R=R_{1}+R_{2}$ in Fig. 3 and so to all systems whether or not they remain bound. The curve with the higher value of $P(i=0)$ corresponds to $\sigma_{\mathrm{k}} / v_{\mathrm{orb}}=1$, and the other one to $\sigma_{\mathrm{k}} / v_{\mathrm{orb}}=2$. These are normalised so that $\int_{0}^{180^{\circ}} P(i) d i=1$. Note that the distribution with the larger velocity spread is more able to produce counter-rotating systems. The dashed lines are the corresponding probability distributions $\left(\sigma_{\mathrm{k}} / v_{\mathrm{orb}}=1\right.$, upper; $\sigma_{\mathrm{k}} / v_{\text {orb }}=2$, lower) with the integral now taken over only the region $R_{2}$ in Fig. [3 and so only including those systems which remain bound. The change in $P(i)$ for given values of $i$ and $\sigma_{\mathrm{k}}$ is due to the fraction of systems which become unbound. The dotted lines are the probability distributions $P(i)$ for systems which remain bound, with $\sigma_{\mathrm{k}} / v_{\text {orb }}=1$, but for which mass loss also occurs. The fractional mass loss is $f=0.2$ for the upper dotted line and $f=0.5$ for the lower.

lower the relative probability of a counter-rotating system and the higher the probability of a system being close to alignment.

\subsection{Bound Systems}

We have calculated the probability distribution of the misalignment angle of the system but have not yet considered whether the resulting system remains bound. If the kinetic energy of the new system exceeds the gravitational potential energy then the system does not remain bound. We no longer have a binary system and the two stars fly apart.

Initially the gravitational energy of the system is

$$
E_{\text {grav }}=-\frac{G M_{1} M_{2}}{a}
$$

and the kinetic energy is

$$
E_{\mathrm{kin}}=\frac{1}{2}\left(\frac{M_{1} M_{2}}{M}\right) v_{\mathrm{orb}}^{2}
$$


in the centre of mass frame, where $M=M_{1}+M_{2}$. In a circular orbit we have

$$
-E_{\mathrm{kin}}=\frac{1}{2} E_{\mathrm{grav}}
$$

and the total energy

$$
E_{\mathrm{tot}}=-\frac{1}{2} \frac{G M_{1} M_{2}}{a} .
$$

So

$$
E_{\text {grav }}=-v_{\text {orb }}^{2} \frac{M_{1} M_{2}}{M} .
$$

After the kick the gravitational energy remains the same because in this case no mass is lost. The kinetic energy becomes

$$
E_{\mathrm{kin}}^{\prime}=\frac{1}{2}\left(\frac{M_{1} M_{2}}{M}\right) v_{\mathrm{n}}^{2}
$$

where $v_{\mathrm{n}}$ is given by equation (2). If $E_{\text {kin }}^{\prime}>-E_{\text {grav }}$ the new system is unbound. The condition for the system to be unbound is $v_{\mathrm{n}}^{2}>2 v_{\mathrm{orb}}^{2}$. We solve $v_{\mathrm{n}}^{2}=2 v_{\mathrm{orb}}^{2}$ with equation (2) for the critical velocity of

$$
v_{\text {bound }}=-\frac{v_{\text {orb }}|\sin \phi|}{\tan i} \pm \sqrt{3 v_{\text {orb }}^{2}+v_{\text {orb }}^{2} \frac{\sin ^{2} \phi}{\tan ^{2} i}} .
$$

Because $v_{\mathrm{k}}>0$ we take the term with the positive sign. If $v_{\mathrm{k}}>v_{\text {bound }}$ then the system is unbound but if $v_{\mathrm{k}}<v_{\text {bound }}$ it remains bound after the supernova kick.

This condition for the system to be bound affects the region in the $v_{\mathrm{k}}-\phi$ plane that we integrate over to find the probability distribution for the misalignment angle. To integrate over all systems, bound or unbound, we integrated equation (18) over the region $R=R_{1}+R_{2}$ shown in Fig. 3 There we also plot the upper limits on the velocity kicks for the system to remain bound as dotted lines, $v_{\text {bound. Below }}$ these lines a system remains bound but above the kick is too strong and the two stars fly apart. The region in which we have bound systems, $R_{2}$, is much smaller than the region that can produce the given misalignment angle, $R_{1}+R_{2}$. To find the probability distribution of the misalignment angle, $P(i)$, for bound systems only we integrate expression (18) over this smaller region $R=R_{2}$.

We compute this numerically and plot it in Fig. 4 as the upper dashed line when $v_{\text {orb }}=\sigma_{\mathrm{k}}$. The lower dashed line is for $v_{\text {orb }}=0.5 \sigma_{\mathrm{k}}$. Most kicks unbind the systems but those that remain bound are somewhat more likely to be counter-aligned than for smaller kicks. On the other hand if $v_{\text {orb }}>\sigma_{\mathrm{k}}$ we find that few kicks are able to cause counteralignment.

As expected the higher the misalignment angle of a system the lower the probability of it forming. We see that, by restricting to only bound systems, the number with small misalignment is greatly reduced whereas those closer to counter-alignment are less so. The probability of a system closer to counter-alignment than alignment becomes relatively high for small $v_{\text {orb }}$.

\subsection{Mass Loss}

So far we have assumed that the mass lost from the system is negligible. We now allow the mass of star 2 to fall in the supernova to

$$
M_{2}^{\prime}=M_{2}-f M
$$

where $f$ is the fraction of mass lost relative to the total mass of the binary system so that the total mass of the system becomes

$$
M^{\prime}=(1-f) M .
$$

The gravitational energy of the system after the supernova is

$$
E_{\text {grav }}^{\prime}=-v_{\text {orb }}^{\prime 2} \frac{M_{1} M_{2}^{\prime}}{M^{\prime}}
$$

where $v_{\text {orb }}^{\prime}$ is the relative velocity that the stars would have in a circular orbit of separation $a$ and

$$
v_{\text {orb }}^{\prime 2}=\frac{G M^{\prime}}{a}=v_{\text {orb }}^{2} \frac{M^{\prime}}{M}=(1-f) v_{\text {orb }}^{2}
$$

because the instantaneous separation $a^{\prime}=a$. Note that $v_{\text {orb }}^{\prime}$ is the orbital velocity for the equivalent circular orbit while the orbit itself is eccentric after the supernova. The new kinetic energy is

$$
E_{\mathrm{kin}}^{\prime}=\frac{1}{2} v_{\mathrm{n}}^{2} \frac{M_{1} M_{2}^{\prime}}{M^{\prime}}
$$

and so the condition for a bound system $\left(E_{\text {kin }}^{\prime}<-E_{\text {grav }}^{\prime}\right)$ becomes

$$
v_{\mathrm{n}}^{2}<2(1-f) v_{\mathrm{orb}}^{2}
$$

Thus we find

$$
v_{\text {bound }}=-\frac{v_{\text {orb }}|\sin \phi|}{\tan i}+\sqrt{(3-2 f) v_{\text {orb }}^{2}+v_{\text {orb }}^{2} \frac{\sin ^{2} \phi}{\tan ^{2} i}} \text {. }
$$

In Fig. 5 we plot contours of $v_{\text {bound }}$ for varying $f$ with $i=$ $0.3 \mathrm{rad}=17^{\circ} .2$ and $v_{\mathrm{orb}}=\sigma_{\mathrm{k}}$. The $v_{+}$and $v_{-}$contours and the top dotted line remain the same as in the top left plot in Fig. 3. The more mass that is lost in the supernova, the lower is the limit on the kick velocity for a bound system and so the less likely a bound system with a given inclination becomes.

In Fig. 4 for $v_{\mathrm{k}}=\sigma_{\mathrm{k}}$ we plot the probability distribution for bound systems for $f=0$ (upper dashed line), 0.2 (upper dotted line) and 0.5 (lower dotted line). A larger $f$ increases the likelihood of counteralignment in bound systems.

\section{ECCENTRICITY PROBABILITY DISTRIBUTION}

In the previous section we discussed the effect of particular supernova kick distributions on the distribution of orbital misalignments. We now consider what kick distributions are most able to give rise to the observed Be-star eccentricity distribution. Given the sparsity of the data, the large number of free parameters and the unknown selection effects, we do not attempt to find a best fit to the periods and eccentricities of Be stars. Rather we look for a kick distribution consistent with these observations and then examine its consequences for the distribution of orbital misalignments.

The new semi-major axis of the orbit after the supernova can be found from

$$
v_{\mathrm{n}}^{2}=G M^{\prime}\left(\frac{2}{a}-\frac{1}{a_{\mathrm{n}}}\right),
$$

where $a$, the old semi-major axis, is the instantaneous separation. Combining this with equation (2) we can find $a_{\mathrm{n}}$. 


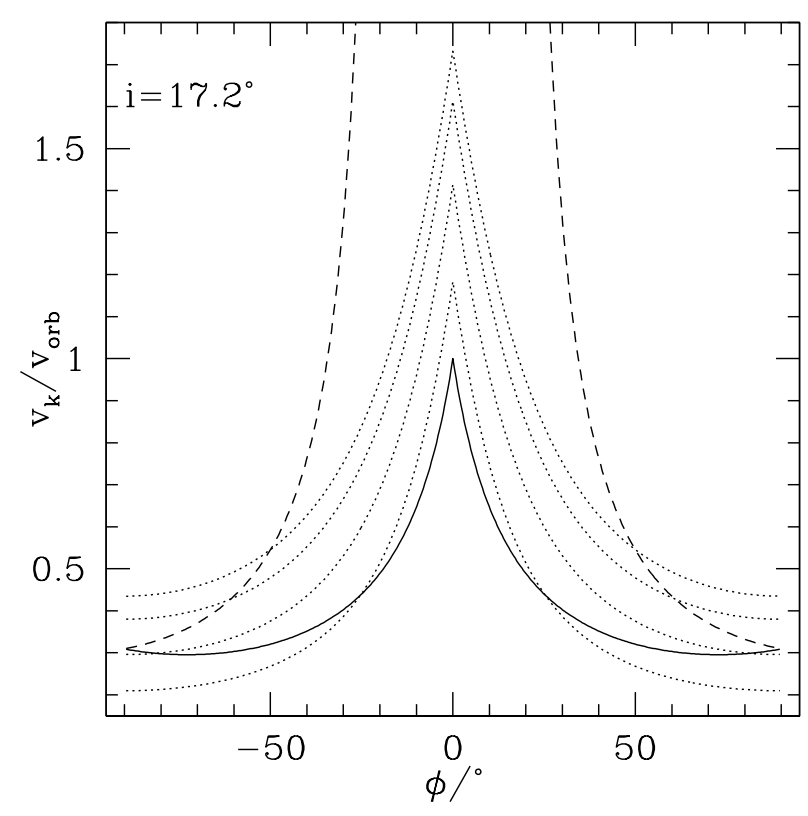

Figure 5. This corresponds to the top left panel in Fig. 3 for $i=17.2^{\circ}$. The solid line and the dashed line are the same in both Figures. The dotted line in Fig. 3 is the same as the uppermost dotted line here and represents the upper boundary for $v_{\mathrm{k}}$ which permits the post-supernova system to remain bound when no mass is lost. When mass is lost from the system the upper limit on $v_{\mathrm{k}}$ such that the system remains bound decreases as the fraction of mass lost $f$ increases. The dotted lines here correspond to $f=0,0.2,0.5$ and 0.8 .

The new system has specific angular momentum

$$
\boldsymbol{h}^{\prime}=\boldsymbol{r} \times \boldsymbol{v}_{\mathbf{n}}
$$

where $\boldsymbol{r}$ is the separation vector of the stars. We have

$$
G M^{\prime} a_{\mathrm{n}}\left(1-e^{2}\right)=\left|\boldsymbol{r} \times \boldsymbol{v}_{\mathbf{n}}\right|^{2}
$$

and so

$$
G M^{\prime} a_{\mathrm{n}}\left(1-e^{2}\right)=a^{2}\left[v_{\mathrm{k}}^{2} \sin ^{2} \phi+\left(v_{\mathrm{k}} \cos \omega \cos \phi-v_{\mathrm{orb}}\right)^{2}\right]
$$

which can be solved to find the eccentricity, $e$, of the new system (Brandt \& Podsiadlowski 1995). The binary system is unbound if $e>1$, in which case $a_{\mathrm{n}} \leqslant 0$. Although we could find eccentricity probabilities by direct integration in a similar way to the inclinations in the previous section, it becomes very complicated and we do not learn much new from the procedure. Instead we use Monte-Carlo methods to evaluate the integrals.

We note that $e$ and $i$ both depend only on $v_{\mathrm{k}} / v_{\text {orb }}$, $1-f$ and the two angles $\phi$ and $\omega$. For typical progenitors of Be stars the dependence on masses, through $v_{\text {orb }}$ and $1-f$ as well as the radius of star 2 , turns out to be weak so it can suffice to concentrate on only one set of masses initially. We choose a pre-supernova mass of $M_{2}=5 M_{\odot}$ that leaves a neutron star of mass $M_{2}^{\prime}=1.4 M_{\odot}$ and a companion mass $M_{1}=15 M_{\odot}$. These masses were used by Brandt \& Podsiadlowski (1995) and we have ensured that we can reproduce their results too.

We use the NAG Library routine G05CAF to generate pseudorandom numbers $\left\{X_{i}\right\}$ uniformly distributed between 0 and 1 . Then for an isotropic kick distribution

$$
\sin \phi=X_{j 1}
$$

and

$$
\omega=2 \pi X_{j 2} .
$$

The distribution of orbital periods, $P_{\mathrm{i}}$, immediately before the supernova depends in a complex way on the previous evolution of the system. There are many as yet unquantified processes that contribute to this evolution (Hurlev, Tout \& Pols 2002) and so we stick with the relatively simple assumption that $\log P_{\mathrm{i}}$ is uniformly distributed between $P_{\min }$ and $P_{\max }$ so that

$$
\log P_{\mathrm{i}}=\log P_{\mathrm{i}}+X_{j 3}\left(\log P_{\max }-\log P_{\min }\right) .
$$

We take $P_{\min }$ to be the period at which star 1 would fill its Roche lobe in a circular orbit if it has the main-sequence radius given by Tout et al. (1996, $5 \mathrm{R}_{\odot}$ for a $15 \mathrm{M}_{\odot}$ star). Its Roche-lobe radius $R_{\mathrm{L}}$ is approximated by the formula of Eggleton (1983),

$$
\frac{R_{\mathrm{L}}}{a}=g(q)=\frac{0.49 q^{\frac{2}{3}}}{0.6 q^{\frac{2}{3}}+\log _{e}\left(1+q^{\frac{1}{3}}\right)}, \quad 0<q<\infty,
$$

where $q=M_{1} / M_{2}$. We take $P_{\max }=10^{3} \mathrm{~d}$ because beyond this almost all systems are disrupted.

For the post-supernova systems Brandt \& Podsiadlowski 1995) rejected any system that would have filled its Roche lobe if it were circular at its periastron separation. So if

$$
(1-e) a_{\mathrm{n}}<\frac{R_{1}}{g(q)}
$$

they rejected the system. In practice we expect that systems cannot actually survive down to this separation because tides enforce pseudosynchronization of star 1 at periastron (Hut 1991) and so it ends up spinning up to about 1.16 times faster than it would in a circular orbit of the periastron separation. However there is no equivalent potential theory in the eccentric orbit so we do not try to be any more precise than condition (42).

We can reproduce figs 4 and 5 of Brandt \& Podsiadlowski (1995). They chose $v_{\mathrm{k}}$ to be constant and used a period distribution which is uniform in $P_{\mathrm{i}}$ rather than $\log P_{\mathrm{i}}$. In their figs $4-6$, for a given $x$-axis value, they found the median on the $y$-axis of 10,000 runs and then worked out the regions in which 20,40,60,80 and $98 \%$ of systems lie away from that median. They found very high values of the mean inclination because they used a high single value kick velocity.

Instead we integrate over $v_{\mathrm{k}}$, distributed according to equation (11) up to $850 \mathrm{~km} \mathrm{~s}^{-1}$ using Simpson's rule and $\omega, \phi$ and $P_{\mathrm{i}}$ by the Monte Carlo method. In our figures we prefer to plot contours of probability density in the $2 \mathrm{D}$ space normalised so that the probability of lying in the plots is 1 .

Rather than sticking to the fixed masses we distribute the masses of the companion star from $M_{1 \min }=5 \mathrm{M}_{\odot}$ to $M_{1 \max }=25 \mathrm{M}_{\odot}$ according to a mass function

$$
N\left(M_{1}\right) d M_{1} \propto M_{1}^{-2.7} d M_{1}
$$

(Kroupa. Tout \& Gilmore 1993) which can be generated 
from

$$
M_{1}=\left(\frac{X_{0}-X_{j 4}}{k}\right)^{-\frac{1}{1.7}},
$$

where we find $X_{0}$ and $k$ from the minimum and maximum masses. Because the mass range is limited the resulting distributions are not very different from the fixed initial masses of $M_{1}=15 \mathrm{M}_{\odot}$ and $M_{2}=5 \mathrm{M}_{\odot}$ as used by Brandt \& Podsiadlowski (1995) to represent a typical Be star binary. Before presenting our results we discuss the observations with which we compare.

\section{MODEL COMPARISON TO OBSERVED SYSTEMS}

There is now a large number of Be star binary systems with measured periods and eccentricities. The data to which we shall apply our models are given in Table1. The bulk of these come from the catalogue of Be/X-ray binaries assembled by Raguzova \& Popov (2005)1. We also include O/B stars that we have found in the literature that have no emission but must have formed in the same way. We can use these to look at the eccentricity distribution and remember that the disc in Be stars may come and go so that B stars can become Be stars and vice versa.

Because we are primarily interested in Be stars with discs we also impose the condition that there must be enough room for a decretion disc of $4 R_{\star}$ or so inside the Roche lobe of star 1 . We explain our choice in the appendix but note that there is one Be star in Table 1 0535-668, that we do not include in our analysis because it cannot accommodate such a disc.

There are three systems for which the misalignment angle $i$ between the Be star spin and disc have been estimated. There is one more system, 28 Tau, which has no reliable orbital parameters but for which an inclination of $i=25^{\circ}-30^{\circ}$ has been suggested.

\subsection{Single Maxwellian Peak Velocity Kick Distribution}

We now consider how well the standard velocity kick distribution of Hobbs et al. (2005) fits the observed data. In the left frame of Fig. 6 we plot the probability distribution contours of eccentricity against final period for these systems and in the right panel we plot contours in the inclination-eccentricity plane. The probability of a highly inclined system is quite small and most systems end up closer to alignment than to counter-alignment. Larger inclinations are more likely for the more eccentric systems.

To illustrate this further, the solid lines in Fig. [7 show the probabilities of $e$ and $i$ integrated over all systems. We also plot a histogram of the observed Be star systems' eccentricities. It is evident, in line with previous findings on less substantial data sets, that the Hobbs et al. (2005) distribution gives a poor fit to the eccentricity distribution. It does, however, give a fairly flat distribution of misalignments. There are too few measured misalignments to use

\footnotetext{
1 http://xray.sai.msu.ru/ raguzova/BeXcat
}

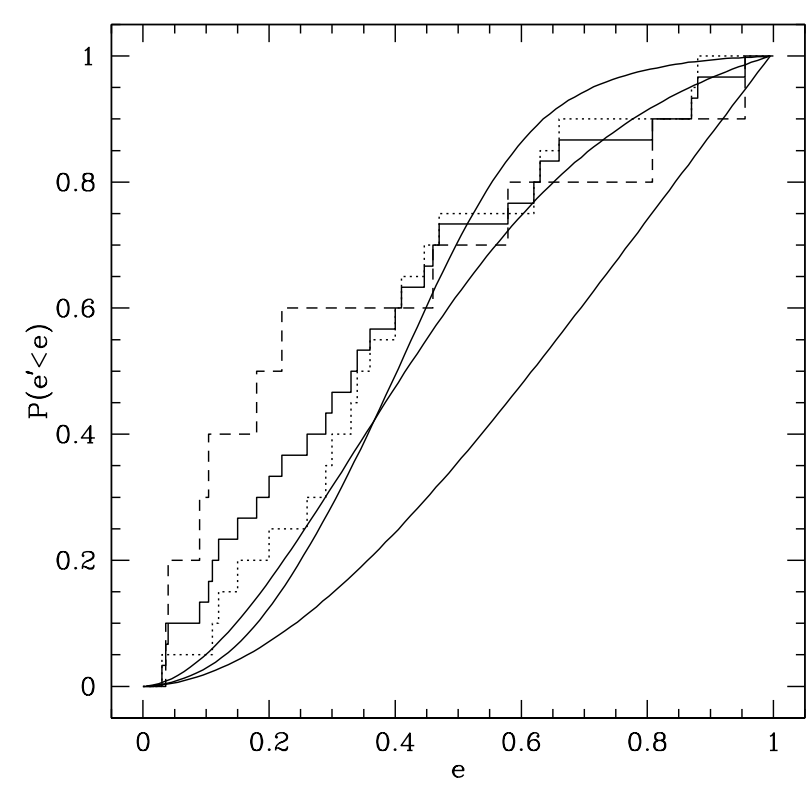

Figure 8. Solid lines are the cumulative eccentricity probability distribution for $\sigma_{\mathrm{k}}=265,15$, and $5 \mathrm{~km} \mathrm{~s}^{-1}$ in order of increasing height on the right hand side in the plot. The cumulative eccentricity probability distribution for the observed systems is shown for the twenty Be stars (dotted line), the ten B stars (dashed line) and for the total of thirty B and Be stars (solid line). We use a KS test to compare the observed distribution of system eccentricities to our model predictions with the results given in Table 2 The quality of fit peaks for the Be stars around $\sigma_{\mathrm{k}}=5 \mathrm{~km} \mathrm{~s}^{-1}$ and for the combined Be and B stars at around $\sigma_{\mathrm{k}}=15 \mathrm{~km} \mathrm{~s}^{-1}$.

these as a test but the measured values of are consistent with the standard Hobbs et al. (2005) kick distribution.

Because the final period distribution depends very strongly on our choice of initial period we have not made use of it for a statistical comparison with observations except to say that the systems appear to fit well in Fig. 6 (and later in Fig. 10). This implies that our choice of period distribution is reasonable.

We use the Kolmogorov-Smirnov test (KS test) to determine if two datasets are significantly different. It is nonparametric and distribution free. In Fig. 8 we plot the cumulative eccentricity distributions of the Be stars, of the B stars and of the B and Be stars combined. We also plot cumulative eccentricity distribution for our model predictions. We then perform a KS test between the observed data and our model prediction and give the results in Table 2 We find the largest deviation of the observed data from the model and use probability tables for the KS test to find the probability that the observed sample of stars came from the distribution predicted by the model. In Table 2 we give the probability that the observations of Be stars, $P_{1}$, B stars, $P_{2}$, and combined $\mathrm{B}$ and Be stars, $P_{3}$ are consistent with our various models. Probabilities smaller than $10^{-4}$ are listed as zero. The very small probabilities for the standard Hobbs et al. (2005) distribution (the first line in Table 2) demonstrates that it is essentially impossible that the B and Be systems have formed as they are in this way, with a single Maxwellian distribution with $\sigma_{\mathrm{k}}=265 \mathrm{~km} \mathrm{~s}^{-1}$.

We cannot, however, immediately rule out a kick distri- 

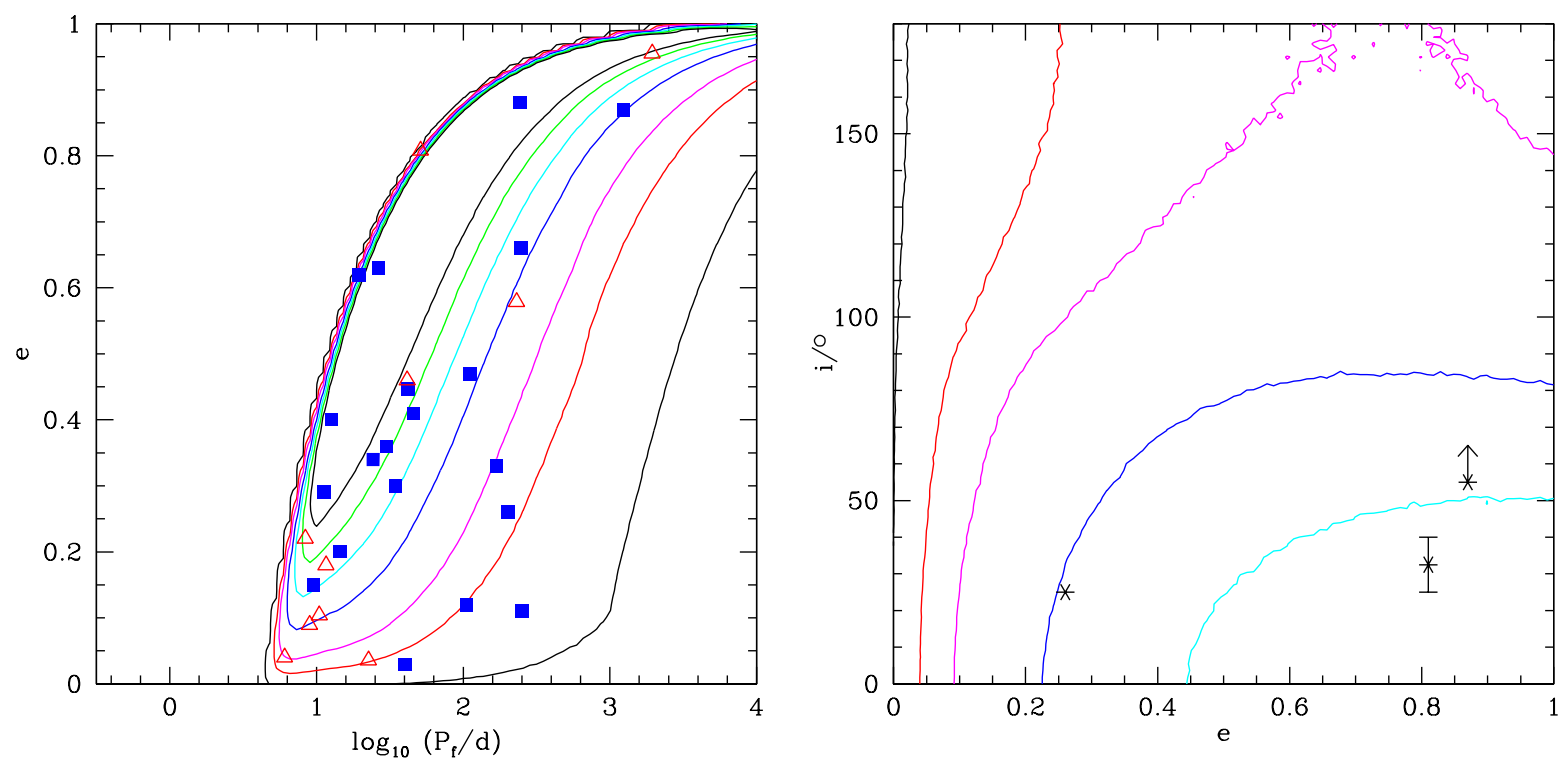

Figure 6. Left: the stars listed in Table 1 in a period-eccentricity diagram. The solid squares are the Be/X-ray binaries and the open triangles are the binary B stars. The contours are lines of constant probability density $P\left(P_{\mathrm{f}}, e\right)$ when the dispersion of the velocity kick distribution in $\sigma_{\mathrm{k}}=265 \mathrm{~km} \mathrm{~s}^{-1}$ and post-supernova binaries which are too tight to permit a disc of size $4 R_{\star}$ are excluded (section 4 ). The probability density $P$ is defined so that the probability of finding a system with $e$ in the interval $(e, e+d e)$ and with period log $P_{\mathrm{f}}$ in the interval $\left(\log P_{\mathrm{f}}, \log P_{\mathrm{f}}+d \log P_{\mathrm{f}}\right)$ is $P d e d \log P_{\mathrm{f}}$. The area at small $P_{\mathrm{f}}$ is excluded by the models. The outermost contour is at $P=0.01$. Moving inwards the contour levels are $P=0.1,0.2,0.4,0.6,0.8$ and 1 . Right: contours of equal probability density $P(e, i)$ for the same models as in the left panel. The contour levels are, starting from the left, $P=0.01,0.1,0.2,0.4$ and 0.6 . The asterisks correspond to the systems given in Table 1 for which there are estimates of the misalignment angle $i$.
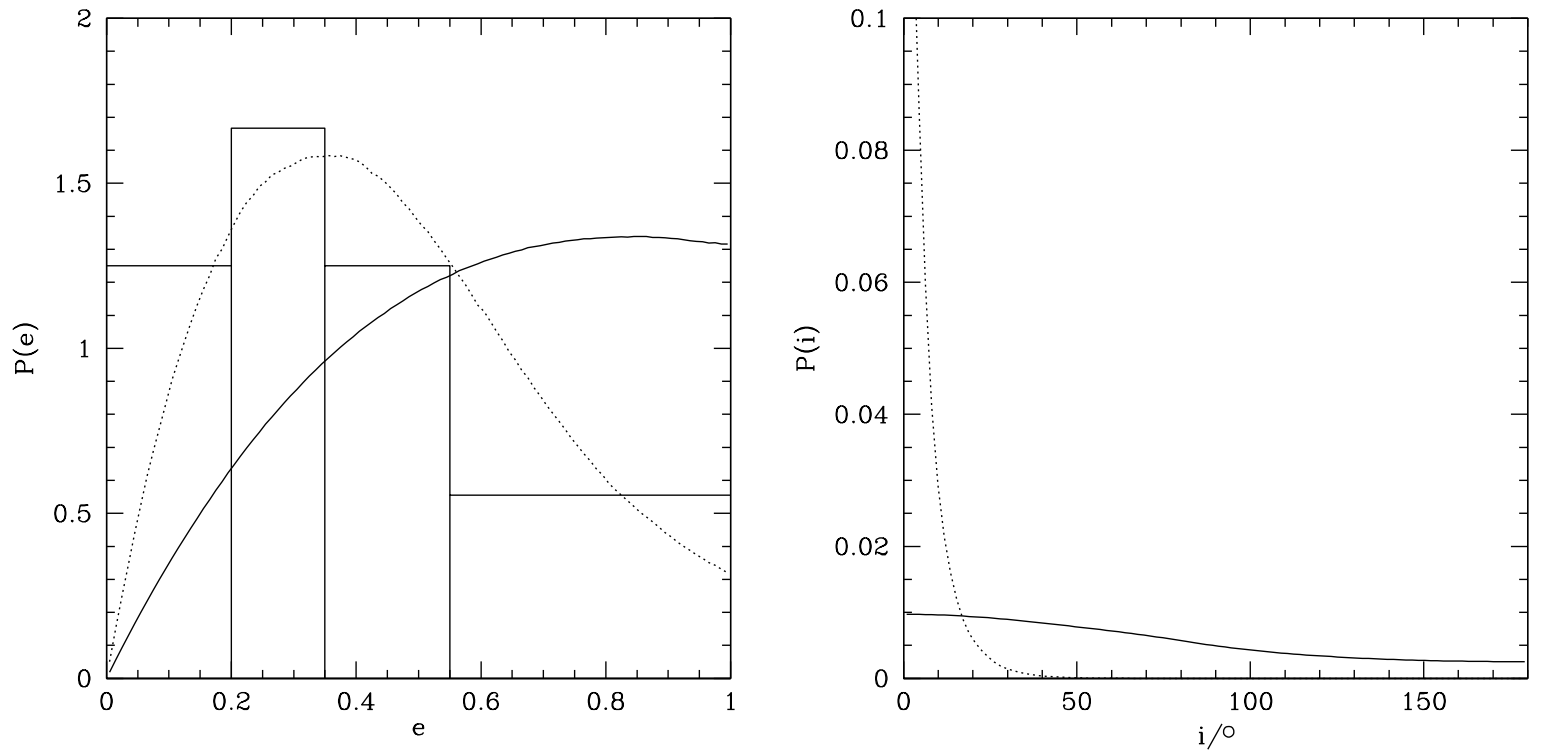

Figure 7. Left: the two dimensional probability density $P\left(P_{\mathrm{f}}, e\right)$ contours, of which are shown in Fig. 66 (left), integrated over period to give a one dimensional probability density $P(e)$. This is plotted as the solid curve (normalised so that $\left.\int_{0}^{1} P(e) d e=1\right)$ and for a kick distribution with $\sigma_{\mathrm{k}}=265 \mathrm{~km} \mathrm{~s}^{-1}$. The dotted line gives the probability density $P(e)$ for models constructed with $\sigma_{\mathrm{k}}=15 \mathrm{~km} \mathrm{~s}{ }^{-1}$. The histogram is the observed eccentricity distribution for the Be stars (the solid squares in Figure 6). The lower value of $\sigma_{\mathrm{k}}$ gives a much better fit to the data. Right: as for the left panel but the probability distributions for misalignment angles $i$ predicted by the models are plotted. The few measured misalignment angles which are known are not plotted but all exceed $i \approx 25^{\circ}$. It is evident that the solid line $\left(\sigma_{\mathrm{k}}=265 \mathrm{~km} \mathrm{~s}^{-1}\right)$ is consistent with a fairly uniform spread of misalignments while the models with $\sigma_{\mathrm{k}}=15 \mathrm{~km} \mathrm{~s}{ }^{-1}$ (dotted line), which provide a better fit to the eccentricity distribution, are not consistent with the observed misalignments. For this model only 7 per cent have $i>15^{\circ}$ and only 1 per cent have $i>25^{\circ}$. 


\begin{tabular}{|c|c|c|c|c|}
\hline & Spectral Type & $P_{\mathrm{f}} / \mathrm{d}$ & $\mathrm{e}$ & $\mathrm{i}$ \\
\hline \multicolumn{5}{|c|}{ Systems with Emission } \\
\hline $0053+604(\gamma$ Cas $)$ & B0.5 IVe & 203.59 & $0.26^{1}$ & about $25^{\circ} 43$ \\
\hline $0115+634$ & $\mathrm{~B} 0.2 \mathrm{Ve}$ & 24.3 & $0.34^{2}$ & \\
\hline 0331+530 (BQ Cam) & O8-9 Ve & 34.3 & $0.3^{3}$ & \\
\hline 0352+309 (X-Per) & O9.5 IIIe-B0 Ve & 250 & $0.11^{4}$ & \\
\hline $0535+262(\mathrm{~V} 725 \mathrm{Tau})$ & B0 III-Ve & 111 & $0.47^{5}$ & \\
\hline $0834-430$ & B0-2 III-Ve & 105.8 & $0.12^{6}$ & \\
\hline J1008-57 & O9e-B1e 38 & 247.5 & $0.66^{40}$ & \\
\hline $1417-624$ & $\mathrm{~B} 1 \mathrm{Ve}$ & 42.12 & $0.446^{7}$ & \\
\hline $1845-024$ & $\mathrm{Be}$ & 242.18 & $0.88^{37}$ & \\
\hline $\mathrm{J} 1946+274$ & B0-1 IV-Ve & 169.2 & $0.33^{9}$ & \\
\hline $\mathrm{J} 1948+32$ & B0 Ve & $40.415 \pm 0.010$ & $0.033 \pm 0.013^{14}$ & \\
\hline $2030+375$ & $\mathrm{~B} 0 \mathrm{e}$ & 46.0202 & $0.416^{10}$ & \\
\hline $\mathrm{J} 2103.5+4545$ & $\mathrm{~B} 0 \mathrm{Ve}$ & $12.66536 \pm 0.00088$ & $0.4055 \pm 0.0032^{11}$ & \\
\hline SAX J0635.2+0533 & B2V-B1IIIe & $11.2 \pm 0.5$ & $0.29 \pm 0.09^{12}$ & \\
\hline XTE J0421+560 & B4 III-V[e] & 19.410 & $0.62^{13}$ & \\
\hline $4 \mathrm{U} 2206+543$ & $09.5 \mathrm{Ve}$ & 9.570 & $0.15^{15}$ & \\
\hline B1259-63 & $\mathrm{Be}$ & 1236.72404 & $0.8698869^{16}$ & greater than $55^{\circ} 42,16$ \\
\hline * 0535-668 & $\mathrm{B} 2$ IIIe $^{35}$ & 16.65 & $0.82 \pm 0.04^{17}$ & \\
\hline 1E 1145.1-6141 & B2Iae & 14.365 & $0.20^{20}$ & \\
\hline GRO J1750-27 & $\mathrm{Be}$ & 29.817 & $0.360^{36}$ & \\
\hline LS I +61 303 & B0 Ve & 26.5 & $0.63 \pm 0.11^{41}$ & \\
\hline \multicolumn{5}{|c|}{ Systems without Emission } \\
\hline $1901+03$ & $\mathrm{OB}$ & 22.58 & $0.036^{8}$ & \\
\hline J0045-7319 & $\mathrm{B}$ & 51.16926 & $0.80798^{18}$ & $25-41^{\circ} 16$ \\
\hline J1740-3052 & $\mathrm{B}^{19}$ & 231.02965 & 0.5788720 & \\
\hline $2 \mathrm{~S} 0114+650(\mathrm{LS} \mathrm{I}+65010)$ & $\mathrm{B} 0.5 \mathrm{Ib}$ & 11.600 & $0.18 \pm 0.05^{21}$ & \\
\hline * $4 \mathrm{U} 1538-52$ & B0 Iab & 3.730 & $0.18 \pm 0.01^{22}$ & \\
\hline $4 \mathrm{U} 1907+09$ & O8-9 Ia & 8.380 & $0.22 \pm 0.05^{23}$ & \\
\hline BP Cru (GX 301-2) & $\mathrm{B} 1.5 \mathrm{Ia}$ & 41.5 & $0.46^{24}$ & \\
\hline OAO $1657-415$ & B0-6 Iab & $10.44809^{25}$ & $0.104^{26}$ & \\
\hline Vela X-1 & B0.5 Ib & 8.964368 & $0.0898^{27}$ & \\
\hline * LMC X-4 & O8 III & 1.40841 & $0.006^{28}$ & \\
\hline $\mathrm{J} 1638-4725$ & & 1940.9 & $0.955^{29}$ & \\
\hline XTE J1855-026 & $\mathrm{O}$ or $\mathrm{B}$ & 6.067 & $0.04 \pm 0.02^{32}$ & \\
\hline
\end{tabular}

Table 1. Binary Be stars and binary B stars that we use in our analysis. We include all the binary B and Be stars we have been able to find reference to in the literature for which both periods and eccentricities are reliably known. Most of the data that we use comes from the catalogue of Be/X-ray binaries assembled by Raguzova \& Popov (2005). We also include measurements or estimates of the misalignment angle $i$ for the few systems for which it is available. The systems marked with an asterisk $(*)$ are not included in our analysis because the stellar separation is not large enough to accommodate a Be-type disc of size $4 R_{\star}$. References for quantities in the Table are given ${ }^{1}$ Harmanec et al. (2000) ${ }^{2}$ Rappaport et al. (1978) ${ }^{3}$ Stella et al. (1985) ${ }^{4}$ Delgado-Martí et al. (2001) $\sqrt[5]{\text { Negueruela et al. (2000) }}{ }^{6}$ Wilson et al. (1997) ${ }^{7}$ Finger. Wilson \& Chakrabarty (1996) ${ }^{8}$ Gallowav, Wang \& Morgan (2005) ${ }^{9}$ Wilson et al. (2003) ${ }^{10}$ Wilson, Fabregat \& Coburn (2005) ${ }^{11}$ Bavkal et al. (2007) ${ }^{12}$ Kaaret. Cusumano \& Sacco (2000) ${ }^{13}$ Barsukova et al. (2007) ${ }^{14}$ Gallowav, Morgan \& Levine (2004) ${ }^{15}$ Ribó et al. (2006) ${ }^{16}$ Hughes \& Bailes (1999) ${ }^{17}$ Hutchings et al. (1985) ${ }^{18}$ Kaspi et al. (1994) ${ }^{19}$ Stairs et al. (2001) ${ }^{20}$ Ray \& Chakrabarty (2002) ${ }^{21}$ Grundstrom et al. (2007a) ${ }^{22}$ Raichur et al. (2006) ${ }^{23}$ Makishima et al. (1984) ${ }^{24}$ van Genderen \& Sterken (2007) ${ }^{25}$ Bildsten et al. (1997) ${ }^{26}$ Chakrabarty et al. (1993) ${ }^{27}$ Bildsten et al. (1997) ${ }^{28}$ Levine et al. (1991) ${ }^{29}$ McLaughlin (2004) ${ }^{32}$ Corbet \& Mukai (2002) ${ }^{35}$ Charles et al. (1983) ${ }^{36}$ Scott et al. (1997) ${ }^{37}$ Finger et al. (1999) ${ }^{38}$ Coe et al. (1994) ${ }^{40}$ Okazaki \& Negueruela (2001) ${ }^{41}$ Casares et al. (2005) ${ }^{42}$ Wex et al. (1998) and ${ }^{43}$ Hummel (1998).

bution in the form of a single Maxwellian distribution with $\sigma_{\mathrm{k}}=265 \mathrm{~km} \mathrm{~s}^{-1}$, because the current eccentricity distribution might not be representative of the eccentricity distribution immediately after the supernova. One possibility is that the systems have begun to circularise by some mechanism but, given that they are not completely circular, the timescale on which this circularisation operates is coincidentally close to the time since the supernova. We return to this in Section 6 A second possibility is that there might be a selection effect on the observed systems. The interaction that leads to emission may only occur close to periastron in the very eccentric systems that spend very little of their time there. Alternatively it may just be that periods and eccentricities are more easily measured for the least eccentric systems. We again return to this in Section 6

On the other hand the observed eccentricity distribution is consistent with a Maxwellian kick distribution provided that the kick distribution is peaked at a lower velocity. In Table 2 we show the effect of reducing $\sigma_{k}$ and find very good fits to all observations when $10<\sigma_{k} / \mathrm{km} \mathrm{s}^{-1}<20$. In Fig. 7 we also plot the dotted lines to show the eccentricity and inclination distributions for a single Maxwellian peak with 


\begin{tabular}{|c|c|c|c|c|c|}
\hline \multicolumn{6}{|c|}{ Single Maxwellian Distribution } \\
\hline$\sigma_{\mathrm{k}}$ & $P_{1}$ & $P_{2}$ & \multicolumn{3}{|l|}{$P_{3}$} \\
\hline 265 & 0.0003 & 0.0025 & 0.0000 & & \\
\hline 190 & 0.0003 & 0.0025 & 0.0000 & & \\
\hline 80 & 0.0013 & 0.0035 & 0.0001 & & \\
\hline 40 & 0.0199 & 0.0088 & 0.0057 & & \\
\hline 20 & 0.1572 & 0.0217 & 0.1000 & & \\
\hline 15 & 0.2550 & 0.0238 & 0.1308 & & \\
\hline 10 & 0.3840 & 0.0213 & 0.1141 & & \\
\hline 5 & 0.4295 & 0.0103 & 0.0445 & & \\
\hline 2 & 0.3699 & 0.0091 & 0.0378 & & \\
\hline \multicolumn{6}{|c|}{ Bimodal Maxwellian Distribution } \\
\hline$\sigma_{\mathrm{k} 1}$ & $\sigma_{\mathrm{k} 2}$ & $w_{1}$ & $P_{1}$ & $P_{2}$ & $P_{3}$ \\
\hline 15 & 265 & 0.4 & 0.2087 & 0.0217 & 0.1168 \\
\hline 15 & 190 & 0.5 & 0.0210 & 0.0088 & 0.0061 \\
\hline 90 & 500 & 0.4 & 0.0009 & 0.0032 & 0.0000 \\
\hline 15 & 500 & 0.4 & 0.2475 & 0.0229 & 0.1279 \\
\hline
\end{tabular}

Table 2. The KS test probability values, the likelihood that the observed systems are chosen from the model distribution. In the upper part of the Table the velocity kick distribution is modelled as a single Maxwellian. In the lower part of the Table the velocity kick distribution is modelled as two Maxwellians with $\sigma_{\mathrm{k} 1}$ and $\sigma_{\mathrm{k} 2}$ and $w_{1}$ the relative weighting of that with $\sigma_{\mathrm{k} 1}$ to that with $\sigma_{\mathrm{k} 2}$. The likelihood for the Be stars only is $P_{1}, \mathrm{~B}$ stars is $P_{2}$ and for the combined $\mathrm{B}$ and $\mathrm{Be}$ stars is $P_{3}$. Probabilities less than $10^{-4}$ appear as zero.

dispersion $\sigma_{\mathrm{k}}=15 \mathrm{~km} \mathrm{~s}^{-1}$. We see that this curve appears to fit the eccentricity distribution much better than that with $\sigma_{\mathrm{k}}=265 \mathrm{~km} \mathrm{~s}^{-1}$. However, for such low velocity kicks, the misalignments tend to be small (right panel of Fig. 7) and are hard to reconcile with the observed values.

\subsection{On Axis Kicks}

It is possible that the direction of the velocity kick in the supernova is restricted (Brandt \& Podsiadlowski 1995). To investigate this we consider the extreme case that the kick is always directed along the spin axis of the star, perpendicular to the orbital plane, so that $\phi=\pi / 2$ and $\omega$ is undetermined. With equation (28) and $v_{\text {orb }}^{2}=G M / a$, we can express equation (34) as

$$
v_{\mathrm{n}}^{2}=v_{\text {orb }}^{2}(1-f)\left(2-\frac{a}{a_{\mathrm{n}}}\right)
$$

and then equation (37) becomes

$$
\begin{aligned}
1-e^{2}= & \frac{1}{1-f}\left(2-\frac{v_{\mathrm{n}}^{2}}{v_{\text {orb }}^{2}} \frac{1}{1-f}\right) \\
& \times\left[\frac{v_{\mathrm{k}}^{2}}{v_{\text {orb }}^{2}} \sin ^{2} \phi+\left(\frac{v_{\mathrm{k}}}{v_{\text {orb }}} \cos \omega \cos \phi-1\right)^{2}\right] .
\end{aligned}
$$

If the kick lies on the $z$-axis we have $\sin \phi=1$ and so the misalignment angle between the old and new orbital planes is

$$
\cos i=\frac{1}{\sqrt{\frac{v_{\mathrm{k}}^{2}}{v_{\mathrm{orb}}^{2}}+1}}
$$

and

$$
v_{\mathrm{n}}^{2}=v_{\mathrm{k}}^{2}+v_{\mathrm{orb}}^{2}
$$

We can then relate the eccentricity and the inclination by

$$
1-e^{2}=\frac{1}{1-f}\left(2-\frac{\sec ^{2} i}{1-f}\right) \sec ^{2} i
$$

and we plot this in the right panel of Fig. 9 For $M_{2}=5 \mathrm{M}_{\odot}$ and $M_{2}^{\prime}=1.4 \mathrm{M}_{\odot}$ we have

$$
1-f=\frac{M^{\prime}}{M}=\frac{1.4+M_{1} / \mathrm{M}_{\odot}}{5+M_{1} / \mathrm{M}_{\odot}} .
$$

For illustration we choose $M_{1}=5,15$ and $25 \mathrm{M}_{\odot}$ and so $f=0.36,0.18$ and 0.12 .

We plot the eccentricity distributions if the kick is parallel to the binary orbital axis in the left panel of Fig. 9. We see that we cannot get highly misaligned systems or loweccentricity systems with a kick in the $z$-direction. The low eccentricities could be explained by circularisation but the highly misaligned systems are ruled out in this case.

\subsection{Bimodal Distribution of Velocities}

A bimodal velocity kick distribution has been suggested to explain both the high-velocity neutron stars and also the fact that neutron stars appear to be easily contained in globular clusters (Katz 1975). The escape velocity of a neutron star from a globular cluster is about $30 \mathrm{~km} \mathrm{~s}^{-1}$ and it is generally believed that about $10 \%$ of neutron stars born within them are retained (Drukier 1996).

Arzoumanian, Chernoff \& Cordes (2002) used observed properties of radio pulsars and other neutron stars to show that a two component velocity distribution fits the data much better than any one component model. They used a distribution of velocities with two Maxwellian distributions

$$
\begin{aligned}
& P\left(v_{\mathrm{k}}\right) d v_{\mathrm{k}} \propto w_{1} \frac{v_{\mathrm{k}}^{2}}{\sigma_{\mathrm{k} 1}^{3}} e^{-v_{\mathrm{k}}^{2} / 2 \sigma_{\mathrm{k} 1}^{2}} \\
&+\left(1-w_{1}\right) \frac{v_{\mathrm{k}}^{2}}{\sigma_{\mathrm{k} 2}^{3}} e^{-v_{\mathrm{k}}^{2} / 2 \sigma_{\mathrm{k} 2}^{2}} d v_{\mathrm{k}},
\end{aligned}
$$

where $\sigma_{\mathrm{k} 1}=90 \mathrm{kms}^{-1}$ and $\sigma_{\mathrm{k} 2}=500 \mathrm{~km} \mathrm{~s}^{-1}$ and $w_{1}=$ $0.4 \pm 0.2$ is the weight of the first distribution. We perform the same KS test on this bimodal distribution and find that it does not fit our eccentricity distribution well at all (see Table 2). We note that almost all systems that fall into the higher peak are disrupted so that the poor fit is entirely due to the high $\sigma_{\mathrm{k} 1}$.

We found previously that the best fitting distribution with one peak had $\sigma_{\mathrm{k}}=15 \mathrm{~km} \mathrm{~s}^{-1}$. For a bimodal distribution with $\sigma_{\mathrm{k} 1}=15 \mathrm{~km} \mathrm{~s}^{-1}$ and $\sigma_{\mathrm{k} 2}=265 \mathrm{~km} \mathrm{~s}^{-1}$ with equal weight we find that the fit is somewhat poorer than for the single peak at $\sigma_{\mathrm{k}}=15 \mathrm{~km} \mathrm{~s}^{-1}$. However it fits the data significantly better than a distribution with just $\sigma_{\mathrm{k}}=265 \mathrm{~km} \mathrm{~s}^{-1}$.

The weight factor $w_{1}$ is unimportant if $\sigma_{\mathrm{k} 2}$ is large enough to disrupt most systems.For $\sigma_{\mathrm{k} 1}=15 \mathrm{~km} \mathrm{~s}^{-1}$ and $\sigma_{\mathrm{k} 2}=500 \mathrm{kms}^{-1}$ with $w_{1}=0.4$ the fit is as good as the single peak at $\sigma_{\mathrm{k}}=15 \mathrm{kms}^{-1}$ (see Table 2). In Fig. 10 we plot the eccentricity-final period and inclinationeccentricity contours for this velocity kick distribution. Most of the surviving systems are from the $\sigma_{\mathrm{k} 1}=15 \mathrm{~km} \mathrm{~s}^{-1}$ part and so the inclinations are all low. Nearly all systems with kicks from $\sigma_{\mathrm{k} 2}=500 \mathrm{~km} \mathrm{~s}^{-1}$ are disrupted. 

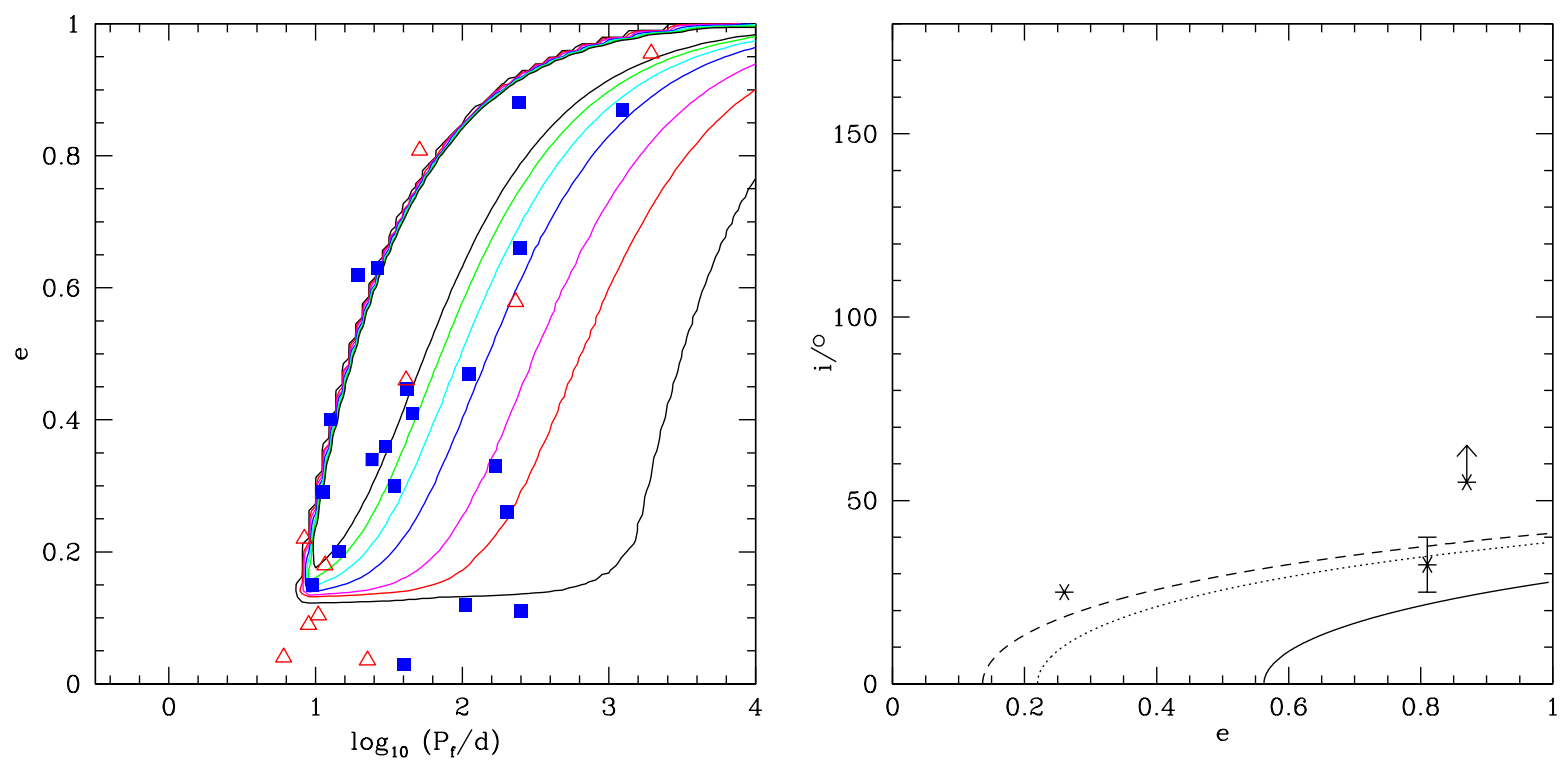

Figure 9. Left: as for Figure 6 except that the direction of the velocity kicks are confined to the $z$-axis (the direction of orbital angular momentum in the pre-supernova binary). As in Figure 6 the contours of probability density range from $P=0.01$ to $P=1$. The solid squares are the Be stars and the triangles are the B stars without emission from Table 1 The models cannot account for the observed low values of the eccentricity in some systems. Right: in this case, because the kick direction is fixed, there is a simple relation between misalignment angle $i$ and eccentricity $e$ for a given fraction $f$ of mass lost in the supernova (equation 49). This relation is plotted for $f=0.12$ (solid line), $f=0.18$ (dotted line) and $f=0.36$ (dashed line). The stars show data for the systems which have estimated misalignment angles.

This bimodal distribution fits the distribution of eccentricities we find here just because the low-kick systems dominate those that remain bound. The effect of the larger kicks is minor simply because most systems with large kicks are disrupted but this would explain the high pulsar space velocities. Until we understand the nature of the supernovae explosions properly in 3-D, bimodal distributions of this kind will remain good but ad hoc solutions.

\section{DISCUSSION}

We have modelled the eccentricity distribution of observed $\mathrm{B}$ and Be stars and we confirm the findings of others that the eccentricities tend to be lower than predicted by a standard Hobbs et al. (2005) kick distribution. The final periods depend on the uncertain choice of inner boundary and initial period distribution. So, to test the models, we compared only the probability distribution of the eccentricities (left panel of Fig. 7). To quantify this we performed a K-S test (see Table 2) to see how well the B and Be stars fit our models. To obtain a good fit from a Maxwellian kick velocity distribution we require $\sigma_{\mathrm{k}}=15 \mathrm{~km} \mathrm{~s}^{-1}$, much lower than the $\sigma_{\mathrm{k}}=265 \mathrm{~km} \mathrm{~s}^{-1}$ of Hobbs et al. (2005). This can be combined with a second Maxwellian as long as that has $\sigma_{\mathrm{k}}$ large enough to disrupt most systems. A combined low and high velocity kicks in the bimodal distribution can then reproduce the high space velocity pulsars and the eccentricity distribution of the bound systems.

In Fig. 7 we show that the few estimates of misalignment angles $i$ between Be star spin axis and disc axis are too large to be easily reconciled with kicks with $\sigma_{\mathrm{k}}$ as low as $15 \mathrm{~km} \mathrm{~s}^{-1}$. They are much better accommodated by $\sigma_{\mathrm{k}}=265 \mathrm{~km} \mathrm{~s}^{-1}$ (Fig. 6). This is even more true for the Bstar binary PSR J0045-7139 in which the B star spins retrogradely with respect to the orbit. However, although the observed misalignments are indicative of high kick velocities, we cannot yet base any firm conclusions on the inclinations because (i) there are only a few, rather uncertain, measurements, (ii) it is easier to measure a large inclination than a small one and (iii) misalignment angles of less than the disc opening angle, estimated to be around $13^{\circ}$ (Hanuschik 1996), would not be sufficient to easily give rise to change between Be star and shell star.

If on the other hand we believe that $\sigma_{\mathrm{k}}$ must be larger, as in the distribution of Hobbs et al. (2005, $\sigma_{\mathrm{k}}=265 \mathrm{~km} \mathrm{~s}^{-1}$ ) and even the bimodal distribution of Arzoumanian, Chernoff \& Cordes 2002, $\sigma_{\mathrm{k} 1}=90 \mathrm{~km} \mathrm{~s}^{-1}$, $\sigma_{\mathrm{k} 2}=500 \mathrm{~km} \mathrm{~s}^{-1}$ ) then we need an alternative explanation of the lower than expected eccentricities. We first consider whether this could be due to observational selection effects. We are less likely to observe systems with high eccentricity if we only see them when they are close to periastron. Such systems spend the majority of their orbital periods closer to apastron and so are less likely to have been observed. In the case of the Be stars this may simply be due to the fact that the companion is much more likely to interact with the decretion disc at periastron. The same would not be true of the plain B stars which appear to follow a similar eccentricity distribution. However there are fewer of them and they still may have been selected by radial velocity variations which would be larger at periastron.

If we assume that we have found all of the Be star systems in our lowest eccentricity bin of $0<e<0.105$ (see 

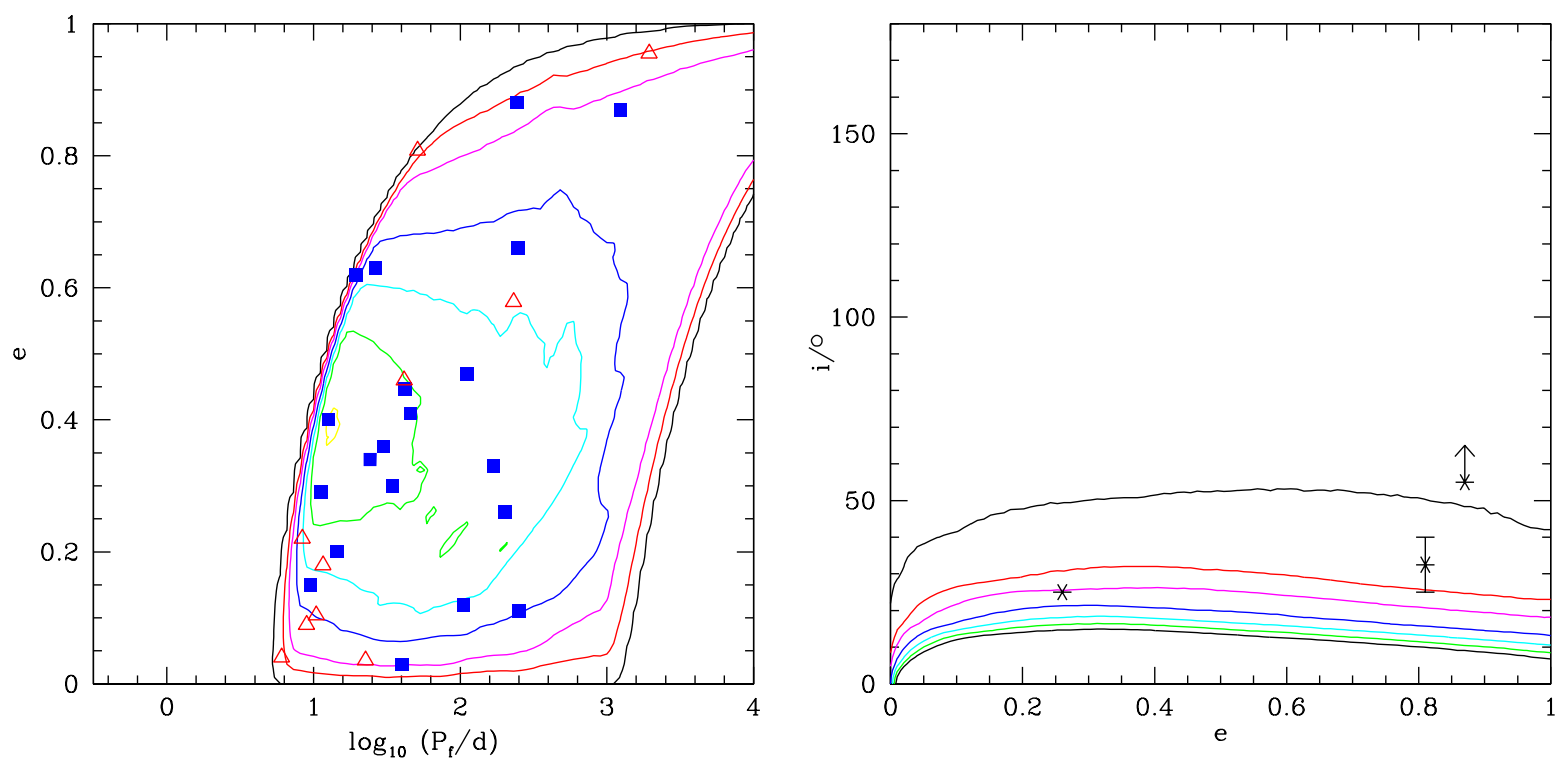

Figure 10. As for Fig. 6 except that the model velocity kick distribution consists of two Maxwellians with $\sigma_{\mathrm{k} 1}=15 \mathrm{~km} \mathrm{~s}{ }^{-1}$ and $\sigma_{\mathrm{k} 2}=500 \mathrm{~km} \mathrm{~s}^{-1}$ and relative fraction of the former $w_{1}=0.4$. The contours in both plots are as in Fig. 6 with contours ranging from $P=0.01$ to $P=1$. Left: the contours of constant probability density in the eccentricity-final period plane. Solid squares are the Be stars and triangles are the B stars without emission in Table 1 By comparison with Fig. 6 it is evident that this model provides a better fit to the observations. This is confirmed by the results of the $\mathrm{K}-\mathrm{S}$ test given in Table 2 Right: the probability density in the $(e, i)$-plane. The asterisks are the systems which have an observed misalignment angle. The observational points are at low values of the probability density, so the observed misalignment angles are hard to reconcile with such a model.

histogram in Fig. 7) then the relative number needs to be brought down by a factor of about 3.4. This would require the actual number of Be star systems in our galaxy to be 3.4 times greater than what we have found and the extra ones must all have high eccentricities. We have found measured eccentricities and periods for 20 Be systems in our galaxy and so would need $3.4 \times 20=68$ systems to account for selection effects. There are 67 observed Be star systems in our galaxy in the Be/X-ray binary catalogue (Raguzova \& Popov 2005) of which 52 do not have both a measured eccentricity and period. It is unlikely that the Be stars without observed eccentricities will all turn out to have high eccentricity. However it is important for observers to measure the eccentricities of more Be star systems because this is vital to rule out selection effects.

Alternatively, and perhaps more interestingly, it is possible that Be stars did form with an eccentricity distribution favouring large eccentricities of $e>0.5$ or so. In this case the systems must have subsequently circularised and the circularisation timescale must be similar to the lifetime of the Be phase. The periods of Be stars can be large and the tidal circularisation time, for these stars with radiative envelopes, is much longer than their lifetimes. One possibility is that the neutron star interacts with the decretion disc of the $\mathrm{B}$ star at periastron passage. Such a mechanism might be self regulating in the sense that the decretion disc only has time to build up to a large radius when the system is very eccentric and the neutron star spends a long time far from the B star. So this merits further investigation particularly if it can be established that supernovae kicks cannot have such a low dispersion of $\sigma_{\mathrm{k}}=15 \mathrm{~km} \mathrm{~s}^{-1}$.

\section{CONCLUSIONS}

The distribution of eccentricities in Be and B stars cannot be reproduced directly if supernova kicks have a Maxwellian distribution with $\sigma_{\mathrm{k}}>30 \mathrm{kms}^{-1}$ or so. Our best fit requires $\sigma_{\mathrm{k}} \approx 15 \mathrm{~km} \mathrm{~s}^{-1}$ though this may be combined to a bimodal distribution with a second Maxwellian with $\sigma_{\mathrm{k} 2} \approx$ $500 \mathrm{~km} \mathrm{~s}^{-1}$, sufficient to disrupt most systems but to account for the high space velocity pulsars. We have also considered the distributions of the misalignment between Be star spin and orbit that would result from such a kick distribution. It is evident that a larger kicks result in larger misalignments. We note that the data indicate that the low-velocity kicks required to give the current eccentricity distribution might not be consistent with the observed misalignments.

If such a low-velocity kick distribution is ruled out then either selection effects must severely limit the observed distribution to such an extent that we are only seeing about two in seven of the high eccentricity systems or the systems must circularise on a timescale similar to their lifetimes. Given that this circularisation is not biased towards low periods, tides in the stars are not sufficient to be its cause in the wide systems. We postulate that a dissipative interaction between the neutron star and the decretion disc is a more likely mechanism. We have suggested that circularisation of Be stars might actually be brought about by an interaction between the neutron star companion and the Be star's decretion disc. This disc is free to grow in size while the neutron star is far away. It spends most of its time at apastron. Then by periastron the disc may have grown sufficiently that the neutron star passes through it and is slowed down. Such an interaction can dissipate energy and thus circularise the or- 
bit. It cannot however change the angular momentum of the star nor alter the inclination of the orbit because both these processes require transfer of angular momentum as well as dissipation of energy. The moment of inertia of the disc is smaller than that of the B star and much less than that of the orbit. The star-disc interaction can therefore easily align the outer parts of the disc with the orbit but any significant change in the angular momentum of the B star or of the orbit can only occur on the disc's viscous timescale. Meanwhile orbital energy can still be dissipated. Thus the misalignment should still contain information on the kick at formation even if the eccentricity no longer does.

Inclinations between the pre- and post-supernova orbits are affected by various factors. Higher kicks lead to fewer bound systems but a larger probability of counteralignment. An increase in the mass lost does the same. Systems with lower pre-supernova periods are less affected and so tend to be more aligned and more able to survive. For the standard Hobbs et al. (2005) kick distribution Be stars ought to be about three times more likely to end up with aligned rather than counter aligned orbits but counteraligned orbits should not be uncommon. On the other hand if $\sigma_{\mathrm{k}}=15 \mathrm{~km} \mathrm{~s}^{-1}$ all orbits should be not far from alignment. In order to distinguish between these various possibilities more information is required about the distribution of misalignment angles between the Be star spin axis and the orientation of the disc.

\section{ACKNOWLEDGEMENTS}

CAT thanks Churchill College for a Fellowship.

\section{REFERENCES}

Arzoumanian Z., Chernoff D. F., Cordes J. M., 2002, ApJ, 568,289

Aschenbach B., Egger R., Trumper J., 1995, Nature, 373, 587

Barsukova E. A., Klochkova V. G., Panchuk V. E., Yushkin M. V., Goranskij V. P., Miroshnichenko A. S., Bjorkman K. S., Manset N., 2007, ATel, 1036, 1

Baykal A., Inam S. Ç., Stark M. J., Heffner C. M., Erkoca, A. E., Swank J. H., 2007, MNRAS, 374, 1108

Bildsten L., Chakrabarty D., Chiu J., 1997, ApJS, 113, 367

Brandt N., Podsiadlowski P., 1995, MNRAS, 274, 461

Casares J., Ribas I., Paredes J. M., Mart J., Allende Prieto C.,2005, MNRAS, 360, 1105

Cassinelli J. P., Brown J. C., Maheswaran M., Miller N. A., Telfer D. C.,2002, ApJ, 578, 951

Chakrabarty D., Grunsfeld J. M., Prince T. A. et al., 1993, ApJ, 403, L33

Charles P. A., Booth L., Densham R. H., Bath G. T., Howarth I. D., Willis A. J., Skinner G. K., Thorstensen J. R., Olszewski E., 1983, MNRAS, 202, 657

Coe M.J., Roche P., Everall C., et al. 1994, MNRAS, 270, L57

Corbet R. H. D., Mukai K., 2002, ApJ, 577, 923

Dachs J., Kiehling R., Engels, D., 1988, A\&A, 194, 167

Delgado-Martí, H., Levine A. M., Pfahl E., Rappaport S., A., 2001, ApJ, 546, 455
Drukier G. A., 1996, MNRAS, 280, 498

Eggleton P. P., 1983, ApJ, 268, 368

Finger M. H., Bildsten L., Chakrabarty D., Prince T. A., Scott D. M., Wilson C. A., Wilson R. B., Zhang S. N., 1999, ApJ, 517, 449

Finger M.H., Wilson R.B., Chakrabarty D., 1996, A\&ASS, 120, 209

Galloway D. K., Wang Z., Morgan E. H. 2005, ApJ, 635, 1217

Galloway D. K., Morgan E. H., Levine A. M., 2004, ApJ, 613, 1164

van Genderen A. M., Sterken C., 2007, IBVS, 5782, 1

Grundstrom, E. D et al, 2007, ApJ, 656, 431

Grundstrom, E. D et al., 2007, ApJ, 660, 1398

Grundstrom E. D., Gies D. R., 2006, ApJ, 651, L53

Gunn J. E., Ostriker J. P., 1970, ApJ, 160, L91

Hansen B. M. S., Phinney E. S., 1997, MNRAS, 291, 569

Hanuschik, R. W., 1996, A\&A, 308, 170

Harmanec P., 2000, A\&A, 364, L85

Hirata R., Active OB-Stars: Laboratories for Stellare and Circumstellar Physics, eds. Okazaki A. T., Owocki S. P., Stefl S., 2007, ASPC, 361, 267

Hobbs G., Lorimer D. R., Lyne A. G., Kramer M., 2005, MNRAS, 360, 974

Hummel W., 1998, A\&A, 330, 243

Hughes A., Bailes M., 1999, ApJ, 522, 504

Hurley J. R., Tout C. A., Pols O. R., 2002, MNRAS

Hut P., 1991, A\&A, 99, 126

Hutchings J. B., Crampton D., Cowley A. P., Olszewski E., Thompson I. B., Suntzeff N., 1985, PASP, 97, 418

Kaaret P., Cusumano G., Sacco B., 2000, ApJ, 542, L41

Kalogera V., 1996, ApJ, 471, 352

Katz J. I., 1975, Nature, 253, 698

Kapsi V. M. et al., 1996, Nat, 381, 584

Kaspi V. M., Johnston S., Bell J. F., Manchester R. N., Bailes, M., Bessell M., Lyne A. G., D'Amico N., 1994, ApJ, 423, L43

Kitaura F. S., Janka H. -Th., Hillebrandt W., 2006, A\&A, 450, 345

Kramer M., Stairs I. H., 2008, ARA\&A, 46, 541

Kroupa P., Tout C. A., Gilmore G., 1993, MNRAS, 262, 545

Lai D., Bildsten L., Kapsi V. M., 1995, ApJ, 452, 819

Lai D., 1996a, ApJ, 466, L35

McLaughlin M., 2004, cxo, prop, 1862

Levine A., Rappaport S., Putney A., Corbet R., Nagase F., 1991, ApJ, 381, 101

Lyne A. G., Lorimer D. R., 1994, Nat, 369, 127

Makishima K., Kawai N., Koyama K., Shibazaki N., Nagase F., Nakagawa M., 1984, PASJ, 36, 679

Morse J. A., Winkler P. F., Kirshnew R. P., 1995, ApJ, 109,2104

Negueruela I., Reig P., Finger M. H., Roche P., 2000, A\&A, 356, 1003

Okazaki A. T., Negueruela I., 2001, A\&A, 377, 161

Pfahl E., Rappaport S., Podsiadlowski P., Spruit H., 2002, ApJ, 574, 364

Podsiadlowski P., Langer N., Poelarends A. J. T., Rappa-

port S., Heger A., Pfahl E., 2004, ApJ, 612, 1044

Porter J. M., 1996, MNRAS, 280, 31

Porter J. M., 1998 A\&A, 336, 966

Pringle J. E., 1996, MNRAS, 281, 357 
Raichur H., Paul B., Naik S., Bhatt N.,2006, AdSpR, 38, 2785

Raguzova, N. V., Popov S. B., 2005, Astron. Astrophys. Transactions, 24, 151

Rappaport S., Clark G. W., Cominsky L., Li F., Joss P. C., 1978, ApJ, 224, L1

Ray P. S., Chakrabarty D., 2002, ApJ, 581, 1293

Ribó, M., Negueruela I., Blay P., Torrejn J. M., Reig P., 2006, A\&A, 449, 687

Rivinius Th., Štefl S., Baade D., 2006, 459, 137

Scheck L., Kifonidis K., Janka H. -Th., Müller E., 2006, A\&A, 457, 963

Scott D. M., Finger M. H., Wilson R. B., Koh D. T., Prince T. A., Vaughan B. A., Chakrabarty D., 1997, ApJ, 488, 831

A. Secchi. Catalogo delle stelle di cui si determinato lo spettro luminoso all' Osservatorio del Collegio romano. Parigi, Per Gauthier-Villars, 1867. Library of Congress Online Catalog.

Shklovskii I. S., 1970, SvA, 13, 562

Sutantyo W., 1978, Ap\&SS, 54, 479

Stairs I. H., 2001, MNRAS, 325, 979

Stella L., White N.E., Davelaar J., et al. 1985, ApJ, 288, L45

Tout C. A., Pols O. R., Eggleton P. P., Han Z., 1996, MNRAS, 281, 257

van den Heuvel, 2007, AIPC, 294, 598

Verbunt F., van der Heuvel E. P. J., ed. Lewin W. H. G. et al., 1995, X-ray Binaries, Cambridge, 457

Wex N., Johnston S., Manchester R. N., Lyne A. G., Stappers B. W., Bailes M., 1998, MNRAS, 298, 997

Wilson C. A., Fabregat J., Coburn W., 2005, ApJ, 620, L99 Wilson C. A., Finger M. H., Coe M. J., Negueruela, I., 2003, ApJ, 584, 996

Wilson C A., Finger M. H., Harmon B. A., Scott D. M., Wilson R. B., Bildsten L., Chakrabarty D., Prince T. A., 1997, ApJ, 479, 388

\section{APPENDIX}

Be stars have a decretion disc around them and theory suggests that Be stars lose their disc to become B stars. If the disc reappears the star becomes a Be star again. If we take this into consideration there must also be room for the decretion disc within the Roche lobe of the B star so we insist that

$$
(1-e) a_{\mathrm{n}}>C \frac{R_{1}}{g(q)}
$$

where $C$ is the size of the disc in units of the stellar radius, to include a system in our analysis. From interferometry Grundstrom \& Gies (2006) find the size of the disc in $\gamma$ Cas to be $R_{\mathrm{d}}=8.1 \pm 1.1 \mathrm{R}_{\star}$ where $R_{\star}$ is the radius of the star so $C=8.1$ and Grundstrom et al. (2007b) find the disc of $\mathrm{X}$ Per to be about six times larger than the stellar radius so $C=6$.

In Fig. 11 we plot the locus defined by equality in equation (52) against $P_{\mathrm{f}}$ for $R_{1} / g(q)=6.9 \mathrm{R}_{\odot}$ for $C=1,2,4$ (solid line), 6, 10 and 20 in the case where the initial mass is the maximum of $M_{2}=25 \mathrm{M}_{\odot}$. For each $C$, the area to the

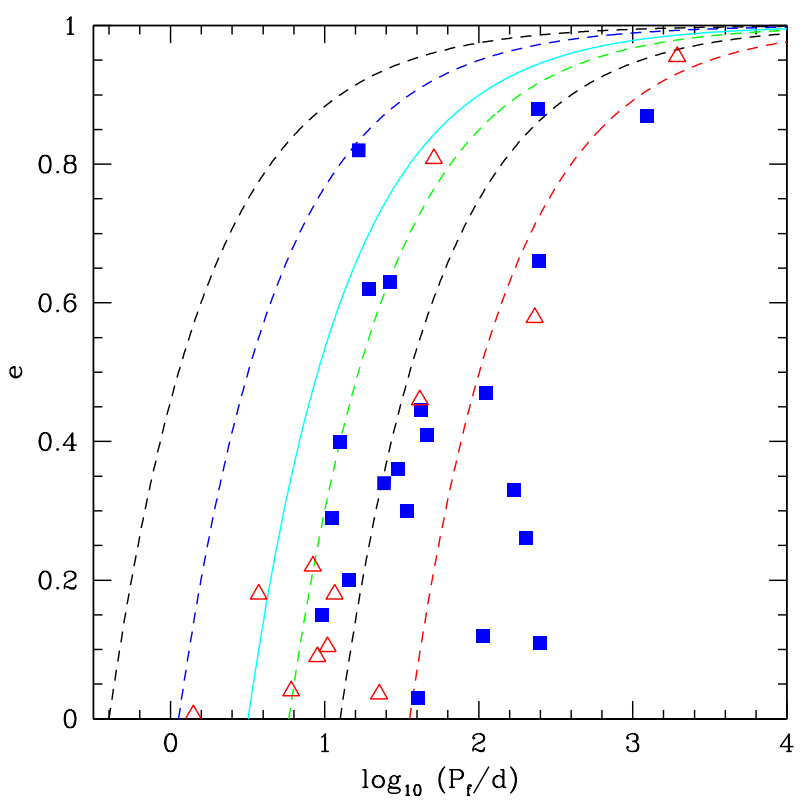

Figure 11. The solid squares are the Be stars and the triangles are the $\mathrm{B}$ stars without emission from table1 The contours show $e=1-a_{\min } / a_{\mathrm{n}}$ against $P_{\mathrm{f}}$ with $C=1,2,4$ (solid line), 6,10 and 20 from left to right. The initial mass of the exploding star is $M_{2}=25 \mathrm{M}_{\odot}$

left of the contour is invalid. Only one Be star system requires $C$ as low as 1.6. This system, $0535-668$ is in the Large Magellanic Cloud which has low metallicity and hence stars have smaller radii. The remainder of Be star systems can be accommodated if $C=4$. The two B stars which lie to the left of the $C=4$ line do not have discs because they show no emission and so we do not include them in our comparisons.

One effect of increasing $C$ from 0 to 4 is to reduce the number of high eccentricity systems at low period. Our initial period distribution biases to low period so this in effect reduces $P(e)$ for large $e$ in what follows. Thus if we were not to include this our conclusions in Section 5.1 would be even stronger. 